\title{
Multitarget, selective compound design yields picomolar inhibitors of a kinetoplastid pteridine reductase 1
}

Ina Pöhner ${ }^{1,2 \dagger}$, Antonio Quotadamo ${ }^{3,4 \dagger}$, Joanna Panecka-Hofman ${ }^{1,5}$, Rosaria Luciani ${ }^{6}$, Matteo Santucci ${ }^{6}$, Pasquale Linciano ${ }^{6}$, Giacomo Landi ${ }^{7}$, Flavio Di Pisa ${ }^{7}$, Lucia Dello lacono ${ }^{7}$, Cecilia Pozzi $^{7}$, Stefano Mangani ${ }^{7}$, Sheraz Gul ${ }^{8}$, Gesa Witt ${ }^{8}$, Bernhard Ellinger ${ }^{8}$, Maria Kuzikov ${ }^{8}$, Nuno Santarem ${ }^{9}$, Anabela Cordeiro-da-Silva ${ }^{9,10}$, Maria Paola Costi $6^{{ }^{*}}$, Alberto Venturelli $i^{3^{\star}}$ and Rebecca C. Wade ${ }^{1,2,11,12^{*}}$

1. Molecular and Cellular Modeling Group, Heidelberg Institute for Theoretical Studies (HITS), D69118 Heidelberg, Germany.

2. Faculty of Biosciences, Heidelberg University, D-69120 Heidelberg, Germany.

3. Tydock Pharma srl, Strada Gherbella 294/B, 41126 Modena, Italy.

4. Clinical and Experimental Medicine PhD Program, University of Modena and Reggio Emilia, 41121 Modena, Italy.

5. Faculty of Physics, University of Warsaw, 02-093 Warsaw, Poland.

6. Department of Life Sciences, University of Modena and Reggio Emilia, Via Campi 103, 41125 Modena, Italy.

7. Department of Biotechnology, Chemistry and Pharmacy, University of Siena, 53100 Siena, Italy.

8. Fraunhofer-IME ScreeningPort, Schnackenburgallee 114, D-22525 Hamburg, Germany.

9. Instituto de Investigação e Inovação em Saúde, Institute for Molecular and Cell Biology, Universidade do Porto, 4200-135 Porto, Portugal.

10. Faculty of Pharmacy, University of Porto, 4050-313 Porto, Portugal.

11. Center for Molecular Biology (ZMBH), DKFZ-ZMBH Alliance, Heidelberg University, D-69120 Heidelberg, Germany

12. Interdisciplinary Center for Scientific Computing (IWR), Heidelberg University, D-69120 Heidelberg, Germany.

Current Addresses: I.P.: School of Pharmacy, University of Eastern Finland, 70211 Kuopio, Finland. †Joint first authors: I.P., A.Q.

"Corresponding Authors: M.P.C., A.V. and R.C.W.

\begin{abstract}
The optimization of compounds with multiple targets is a difficult multidimensional problem in the drug discovery cycle. Here, we present a systematic, multidisciplinary approach to the development of selective anti-parasitic compounds. Computational fragment-based design of novel pteridine derivatives along with iterations of crystallographic structure determination allowed for the derivation of a structure-activity relationship for multitarget inhibition. The approach yielded compounds showing picomolar inhibition of $T$. brucei pteridine reductase 1 (PTR1), nanomolar inhibition of L. major PTR1, and selective submicromolar inhibition of parasite dihydrofolate reductase (DHFR) versus human DHFR. Moreover, by combining design for polypharmacology with a property-based on-parasite optimization, we found three compounds that exhibited micromolar $\mathrm{EC}_{50}$ values against $T$. brucei brucei, whilst retaining their target inhibition. Our results provide a basis for the further development of pteridinebased compounds, and we expect our multitarget approach to be generally applicable to the design and optimization of anti-infective agents.
\end{abstract}

\section{Keywords}

Kinetoplastid; antiparasitic inhibitors; folate pathway enzymes; structure-based drug design; virtual screening; microwave assisted organic synthesis; structure-activity relationships; polypharmacology 


\section{Introduction}

The World Health Organization has identified 17 Neglected Tropical Diseases (NTDs) that pose a health burden to over 1.4 billion people. ${ }^{1,2}$ Parasites of the trypanosomatid family are responsible for two potentially lethal insect-vector borne NTDs: Human African Trypanosomiasis (HAT, sleeping sickness), caused by Trypanosoma brucei, and leishmaniasis, caused by the intracellular parasite Leishmania spp. ${ }^{3-7}$ Current therapeutics are limited by toxicity, poor efficacy and parasite resistance, thus underlining the need for new chemotherapies. ${ }^{8,9}$

New anti-parasitic agents can be identified by target-based drug design strategies. ${ }^{10-12}$ The folate pathway enzyme dihydrofolate reductase (DHFR) is a known anti-cancer, anti-bacterial and anti-malarial target. ${ }^{13-16}$ It provides reduced folates, which are crucial to biological processes like DNA, protein and amino acid synthesis or one-carbon transfer. ${ }^{14,17,18}$ In trypanosomatids, DHFR inhibition, for example by methotrexate (MTX, 1a), is ineffective due to a metabolic bypass via the biopterin-reducing pteridine reductase 1 (PTR1, Figure 1): when DHFR is inhibited, PTR1 is overexpressed and sustains sufficient reduced folate levels to ensure parasite survival. Thus, when targeting the folate pathway in Leishmania, both DHFR and PTR1 need to be considered. ${ }^{19-21}$ In T. brucei, RNA interference studies have suggested PTR1 to be a potential anti-parasitic target in its own right. ${ }^{22,23}$ Nonetheless, even nanomolar PTR1 inhibitors have so far shown limited anti-parasitic activity in vitro ${ }^{24,25}$, suggesting that targeting the T. brucei folate pathway may also benefit from the consideration of both PTR1 and DHFR.

Screening a set of folate-related compounds against parasitic folate pathway targets previously led to the identification of compounds $1 \mathbf{b}$ (methyl-1-(4-(((2,4-diaminopteridin-6-yl) methyl) (methyl) amino) benzoyl) piperidine-4-carboxylate) and 1c (methyl-1-(4-(((2,4diaminopteridin-6-yl)methyl)amino)benzoyl)piperidine-4-carboxylate) as submicromolar inhibitors of Leishmania major PTR1 (LmPTR1) with $\mathrm{K}_{i}$ values of $0.04 \mu \mathrm{M}$ and $0.10 \mu \mathrm{M}$, respectively. ${ }^{26} 1 \mathrm{c}$ was additionally a micromolar inhibitor of $L$. major DHFR ( $\left.L m D H F R\right)$ with a weak selectivity for the parasite enzyme over the human DHFR (hDHFR) (Ki of $4 \mu \mathrm{M}$ vs. 10 $\mu \mathrm{M})$. In contrast to the parasite DHFR, which is covalently coupled with thymidylate synthase (TS) in a bifunctional DHFR-TS, the hDHFR off-target is monofunctional and shares only about $30 \%$ sequence identity with parasite DHFR domains, indicating potential for further optimization of selectivity. ${ }^{27-29}$

The current study focuses on optimizing pteridine-based compounds for their inhibition of $T$. brucei PTR1 (TbPTR1) and TbDHFR, in addition to the corresponding Leishmania targets, while ensuring selectivity against the off-target hDHFR. The enzymatic evaluation of reference pteridines reported earlier ${ }^{26,30}$, our comparative study of trypanosomatid folate pathway proteins $^{31}$, and computational docking studies were first employed for the design of novel 
pteridine derivatives. Three new crystal structures of complexes of pteridines with TbPTR1 and a complex with LmPTR1 were determined and confirmed the predicted bound orientation of the novel pteridines. A systematic analysis of correlations between computed physicochemical molecular descriptors and observed anti-parasitic effects was then performed and allowed us to prioritize promising compounds for synthesis. In total, we identified 26 new pteridine-based multitarget inhibitors showing improved target inhibitory profiles for PTR1 and DHFR of both L. major and T. brucei. Among these inhibitors, we report the first, to the best of our knowledge, picomolar inhibitors of TbPTR1 and several new low nanomolar inhibitors of LmPTR1, which mostly also show selective micromolar to submicromolar inhibition of the parasite DHFR variants. In vitro evaluations of the designed multi-target inhibitors against bloodstream forms of $T$. brucei brucei revealed low micromolar to submicromolar $\mathrm{EC}_{50}$ values for three of these pteridines. Taken together, we here report a successful application of systematic multitarget design approach to yield selective pteridinebased anti-parasitic compounds affecting multiple trypanosomatid enzymes.

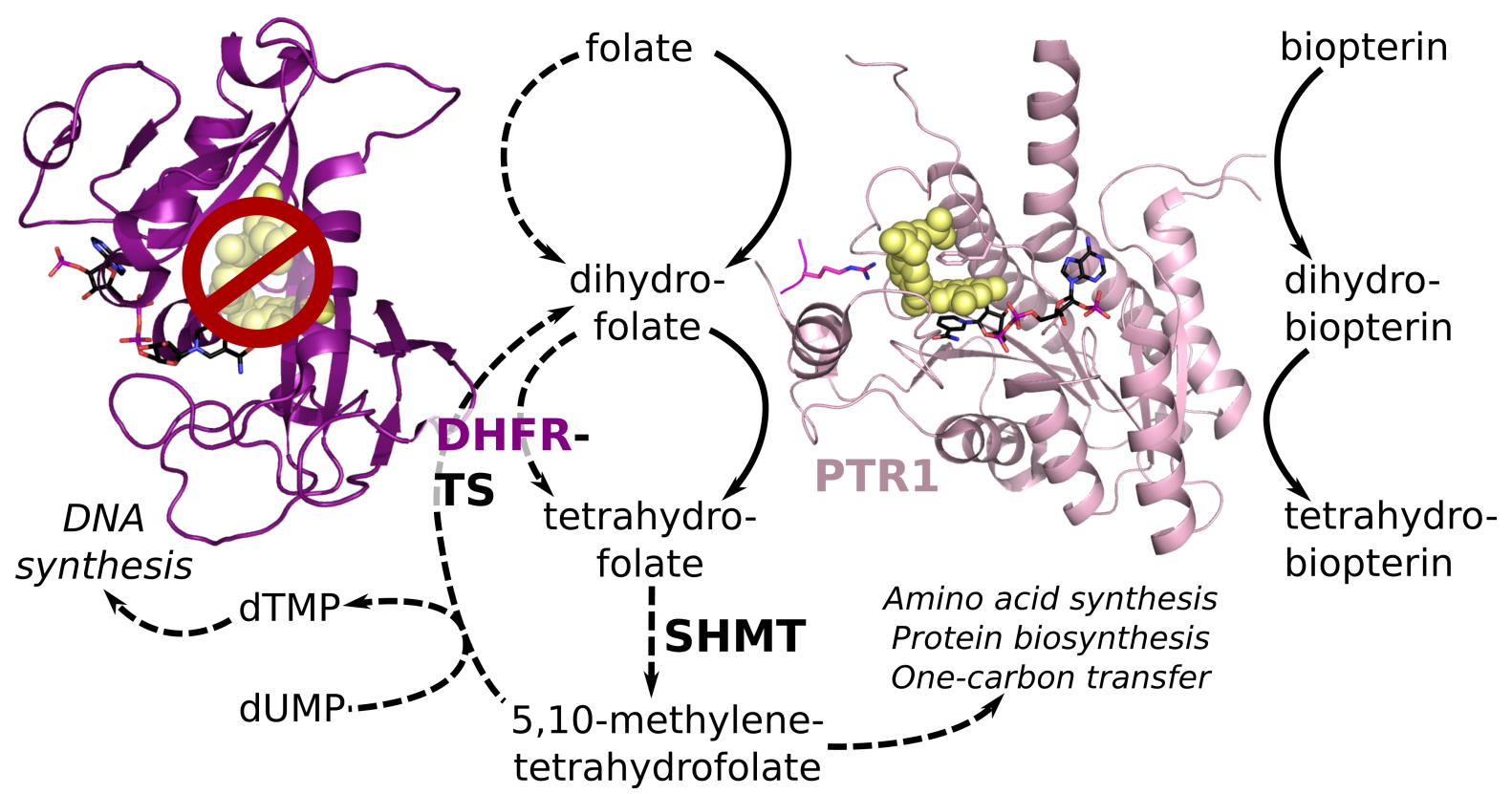

Figure 1. Overview of pterin activation in the trypanosomatidic folate pathway when DHFR is inhibited and PTR1 provides a metabolic bypass. Under normal conditions (indicated by dashed lines), the DHFR domain of the bifunctional DHFR-TS reduces biological folates to tetrahydrofolate (THF). Serine hydroxymethyl transferase (SHMT) converts THF to 5,10-methylene THF, which has a central role in amino acid synthesis, protein biosynthesis and one-carbon transfer. It is also required by the TS domain of DHFR-TS to convert deoxyuridine monophosphate (dUMP) to deoxythymidine monophosphate (dTMP), which is necessary for DNA synthesis. PTR1 catalyzes the reduction of unconjugated pterins, like biopterin, and takes over folate reduction when DHFR is inhibited (continuous lines), thus acting as a metabolic bypass and an important additional target for shutting down the trypanosomatidic folate pathway. Both proteins are shown in cartoon representation (DHFR domain of DHFRTS: purple, PTR1 monomer of the functional tetramer: light pink) with the NADPH/NADP ${ }^{+}$cofactor in stick representation with black carbons and the folate substrate in yellow spheres. In PTR1, an arginine residue from a neighboring subunit that points into the active site is shown in magenta stick representation. 


\section{Results and discussion}

\section{Reference compounds inhibit both PTR1 and DHFR}

To systematically assess multitarget inhibition, we measured the inhibition of TbPTR1, TbDHFR, LmPTR1, LmDHFR, and the off-targets hDHFR and hTS, by the folate-related anticancer agent methotrexate (MTX, 1a) and 7 further pteridine-based reference compounds (1b-h, Figure 2 and Table S1, SI). ${ }^{26,30,32}$ Although $\mathbf{1 b}$-1 $\mathbf{h}$ were primarily designed as LmPTR1 inhibitors, we found all except $\mathbf{1 h}$ to be more potent against TbPTR1 than LmPTR1 with $\mathbf{1 b}$ being the strongest inhibitor of TbPTR 1 with an $\mathrm{IC}_{50}$ of $50 \mathrm{nM}$ against TbPTR1 and $0.3 \mu \mathrm{M}$ against LmPTR1 (Figure 2A). Notably, these compounds exhibited micromolar to submicromolar inhibition of $L m D H F R$ and TbDHFR (IC 50 LmDHFR 0.3-1.4 $\mu$ M; TbDHFR 1.0 $-22.9 \mu \mathrm{M})$. While MTX (1a) was more potent against the parasite DHFRs, it was not selective (Selectivity index SI: TbDHFR/hDHFR $=3$ and $L m D H F R / h D H F R=1$, Figure 2A). For compounds $\mathbf{1 b}-1 \mathrm{~h}$, higher SI values were observed, ranging up to about 165 for $\mathbf{1 b}$ for both TbDHFR and LmDHFR (Figure 2A).

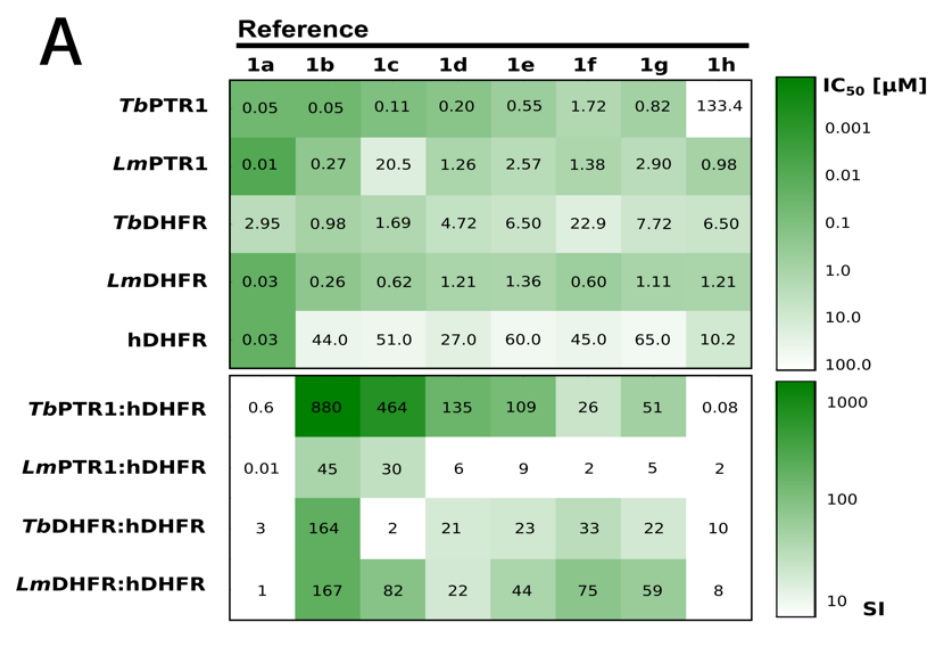

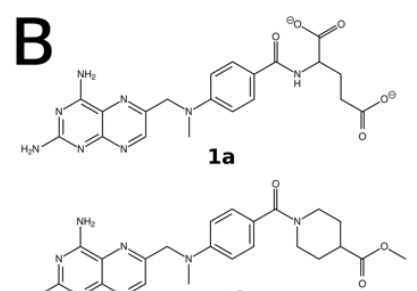

1b

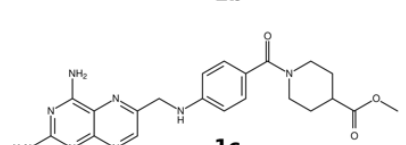

1c

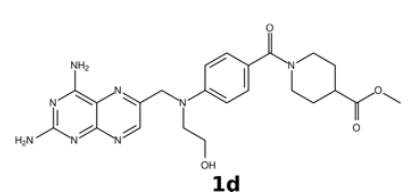

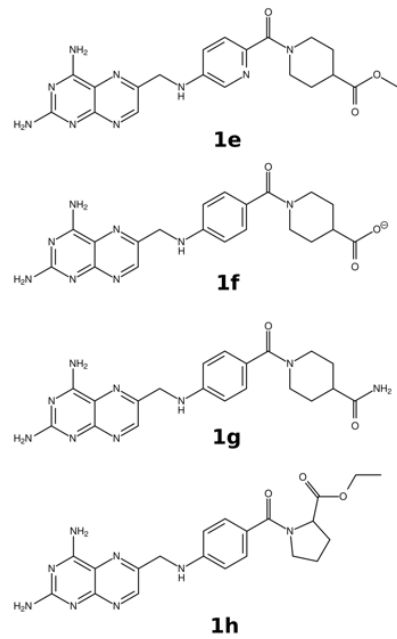

1h

Figure 2. Inhibitory activities, selectivities and structures of reference pteridines. (A) The heatmaps show activities given by IC 50 values (top) and selectivity indices (SI) (bottom) for the targets and the off-target hDHFR. All values, as well as data for hTS, are given in Table S1. (B) The previously published compounds shown were used as reference compounds: $\mathbf{1 a}$ is methotrexate; $\mathbf{1 b}, \mathbf{1 c}$ and $\mathbf{1 h}$ are $\mathbf{6 b}, \mathbf{6 a}$ and $\mathbf{6 c}$ from Cavazzuti et al. ${ }^{26}$ and $\mathbf{1 d}, \mathbf{1 e}, \mathbf{1 f}$ and $\mathbf{1 g}$ correspond to $\mathbf{5 d}, \mathbf{5 b}, \mathbf{6 a}$ and $\mathbf{5 a}$ from Corona et al. $^{30}$

\section{Substrate-like and methotrexate inhibitor-like binding modes of the reference compounds}

Despite the hydrogen-bonding network stabilizing the pteridine ring in the PTR1 active site, for the PTR1 complexes with MTX/1a derivatives, there are two alternative binding modes. Previously determined crystal structures show that compounds $\mathbf{1 b}$ and $1 \mathrm{c}$ share a substratelike pterin orientation in the complex with $L m P T R 1 .{ }^{26}$ In the same crystal structure, compound $\mathbf{1 b}$ also adopts a second, so-called inhibitor-like (or MTX-like) orientation, with the bicyclic ring system flipped by $180^{\circ}$ and rotated by $30^{\circ}$ (Figures $3 \mathrm{AB}$ and S1). ${ }^{26}$ Dual binding modes have 
also been observed in crystallographic complexes of TbPTR1 with small pteridine-based inhibitors. $^{32}$

We here determined the crystal structure of the ternary complex of TbPTR1 with $\mathrm{NADPH} / \mathrm{NADP}^{+}$and the reference compound 1b (PDB-ID 6rx5, resolution $1.42 \AA$, experimental details: Tables S2-S3). It shows that the diaminopteridinyl moiety of $\mathbf{1 b}$ adopts
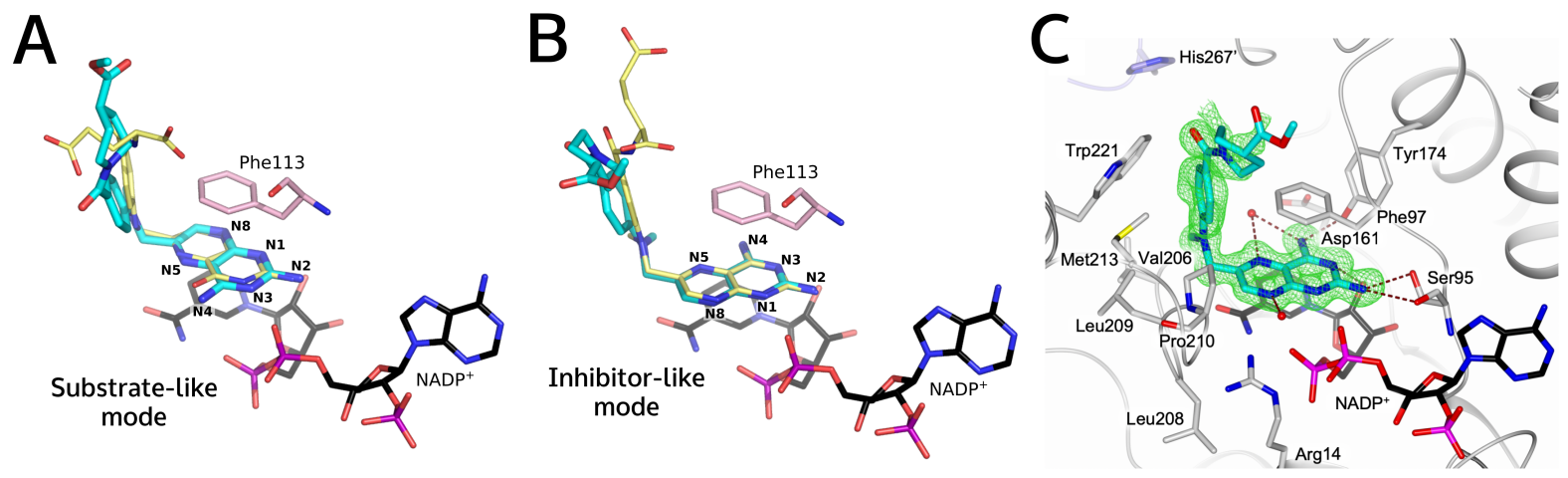

Figure 3. Orientations of reference pteridine compound $1 \mathbf{b}$ in crystal structures of LmPTR1 and TbPTR1. (A,B) Compound 1b (cyan carbons) in complex with LmPTR1 (PDB-ID 2qhx) has a substrate-like $(\mathrm{A})$ and an inhibitor-like or MTX-like (B) binding mode. $\mathbf{1 b}$ is shown with $(A)$ folate (yellow carbons) superimposed from aTbPTR1 structure (PDB-ID 3bmc) and with (B) MTX (1a, yellow carbons) superimposed from a LmPTR1 structure (PDB-ID 1e7w). The pteridine nitrogens are labeled according to the ring nomenclature. $(\mathrm{C})$ The binding site in the crystal structure determined in this work (PDB-ID 6rx5) of TbPTR1 (gray cartoon, His267' from the neighboring subunit in lavender) in complex with NADPH/NADP ${ }^{+}$and compound $1 \mathbf{b}$, which has the MTX-like binding mode. Interacting residues (in A, B: only Phe113) and the NADPH/NADP ${ }^{+}$cofactor are shown in sticks (carbons colored according to protein and black, respectively). In (C), water molecules are shown as red spheres and the inhibitor is surrounded by the omit map (green wire) contoured at the $2.5 \sigma$ level. Hydrogen bonds are represented by brown dashed lines.

only the MTX-like orientation (Figure $3 \mathrm{C}$ ), resembling its MTX-like binding mode in LmPTR1 (Figures 3B and S2). Consistently, docking studies indicated that all reference pteridines adopt MTX-like binding modes in the different targets and the off-target hDHFR (Table S4, Figure S5). Therefore, we concluded that the MTX-like binding mode is likely the dominant one and we focused on the analysis of this binding mode in the subsequent compound design.

\section{Comparative target/off-target mapping and docking studies support design focused on selective multitarget inhibition}

To develop enhanced selective inhibitors of the parasite targets, we employed a multitargetbased design approach to improve inhibition of TbPTR1, TbDHFR, LmPTR1 and LmDHFR, while retaining low hDHFR off-target inhibition. The next generation of pteridine-like compounds was created by dissecting the part of $\mathbf{1 b}$ attached to the pteridine core into three modules. These were: N10, the substitution to the N10 position; PABA, the para-amino benzoic acid (PABA) moiety; and Tail, the cyclic glutamate tail (Figure 4). We separately modified each of these modules to obtain three new series of compounds. The modifications of each module were based on binding mode predictions from docking in the different targets 
and the off-target hDHFR and our previously published optimization guidelines for MTX-like scaffolds. ${ }^{31}$ The key concepts adopted in the compound design are summarized in Figure 4.

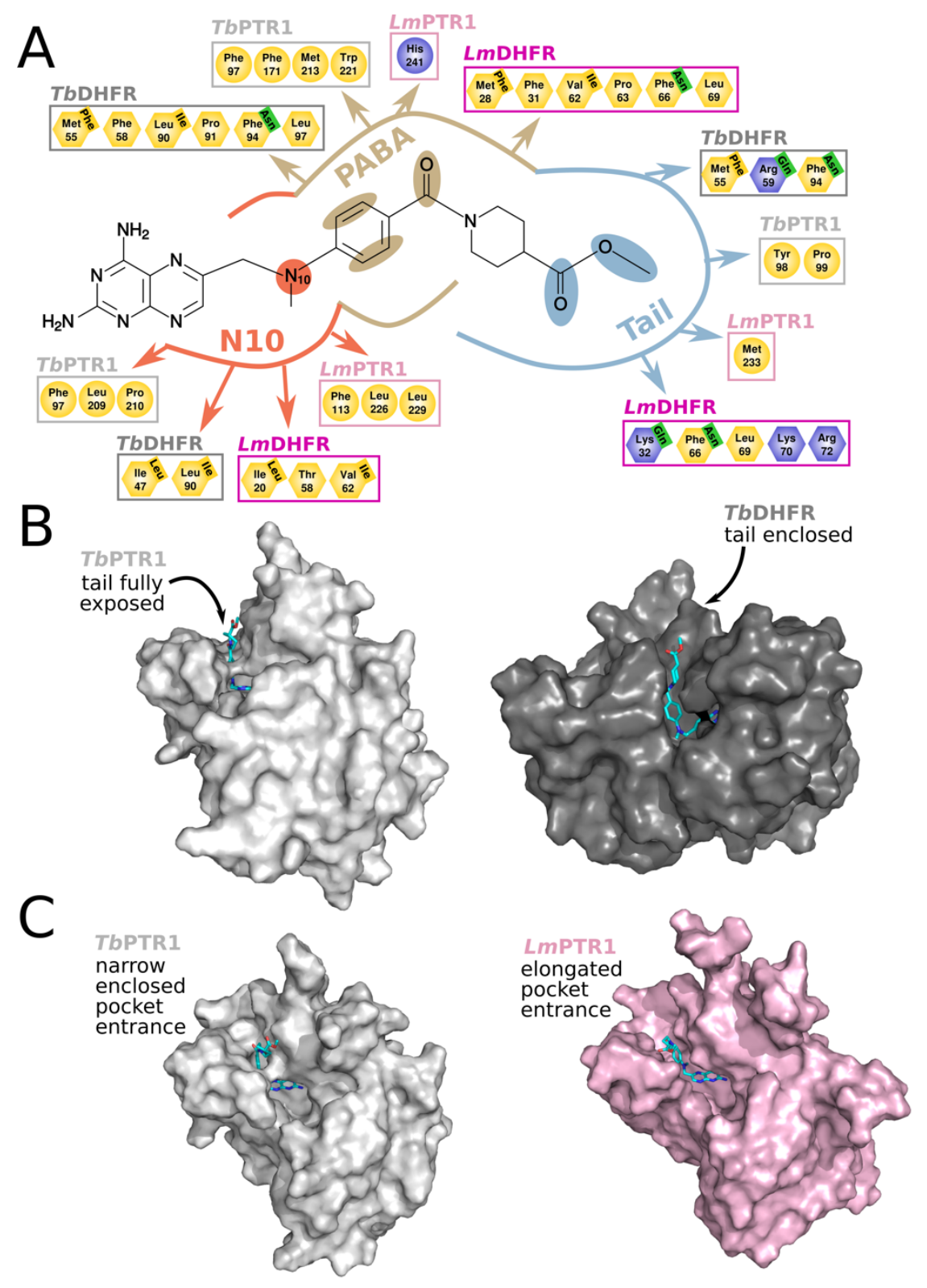

Figure 4. Structural features of PTR1 and DHFR considered in the multitarget design of selective compounds illustrated for reference compound 1b. (A) Selected residues within $5 \AA$ of the three modules - N10, PABA and Tail - modified in the design procedure. Residues were selected for the complexes of 1b with TbPTR1 (pale gray), TbDHFR (dark gray), LmPTR1 (pale pink) and LmDHFR (dark pink). Residues are colored according to their properties: basic: blue, polar: green, and nonpolar: yellow. The ligand interaction plot is based on Panecka-Hofman et al. ${ }^{31}$ and provides an overview of residues with similar properties that surround the ligand modules in the different targets (showing only those applied for the design, for full maps, see Figures S3, S4). In some positions, the amino acid type of the off-target hDHFR is different from parasite DHFR. Differing hDHFR residues are labeled in the top right corner of the corresponding parasite DHFR residue. These positions highlight suitable substitution points to improve selectivity. (B) Surface representations of complexes of 1b with TbPTR1 (left, PDB-ID 6rx5) and TbDHFR (right, MTX-like top-ranked docking pose in PDB-ID 3rg9). The compound tail moiety is fully solvent-exposed in PTR1, whereas it is well-enclosed in DHFR. (C) Surface representations of complexes of 1b with TbPTR1 (left, PDB-ID 6rx5) and LmPTR1 (right, PDB-ID 2qhx, state A). The ligand is more enclosed in the narrow pocket entrance of TbPTR1, while the LmPTR1 pocket has an elongated, widened funnel that can accommodate larger compound tails. In (B, C), $\mathbf{1 b}$ is shown in sticks with cyan carbons. 


\section{Rationale for N10 modifications}

The binding pockets of the different target proteins were found to share a number of aliphatic residues in the proximity of the N10 substituent of a bound ligand, e.g. Leu209 of TbPTR1; Ile47 and Leu90 of TbDHFR; Leu226 and Leu229 of LmPTR1; Ile20 and Val62 of LmDHFR (Figure 4A). ${ }^{31}$ Bulkier nonpolar groups in comparison to the methyl of $\mathbf{1 b}$, like the ethyl and propargyl substituents of $\mathbf{2} \mathbf{a}$ and $\mathbf{2} \mathbf{b}$, allow for interactions with those hydrophobic moieties. Docking studies suggested that even substituents of the size of benzyl, as in $\mathbf{2 c}$, can be accommodated in the PTR1 and DHFR pockets (Figure 5AB). Furthermore, such bulky substituents may improve selectivity for the on-targets: The hDHFR pocket has a lower volume compared to the parasite DHFR pockets (pocket volume TbDHFR $353 \AA^{3}, L m D H F R 384 \AA^{3}$ and hDHFR $347 \AA^{3}$ ).

Furthermore, as previously demonstrated ${ }^{31}$, hDHFR favors hydrogen bond donors in the proximity of $\mathrm{N} 10$ and the PABA ring system, whereas the parasite DHFRs allow for favorable interactions with hydrogen bond acceptors. To improve off-target selectivity, we thus replaced $\mathrm{N} 10$ by sulfur and the PABA benzene ring by pyridine in $\mathbf{2} \mathbf{d}$.

Although Corona et al. ${ }^{30}$ found improved selectivity for PTR1 over hDHFR by hydrophilic N10 substitutions, our data for reference compound 1d with a hydroxyethyl substituent did not support this observation (Figure 2A). Docking simulations indicated that interactions with a highly conserved structural water might induce an unfavorable conformation of the substituent's aliphatic chain (Figure S5A, SI). To relax the geometry while allowing interactions between the substituent and water, we elongated the aliphatic linkage to a hydroxypropyl in 2e.

Thus, in total, the N10 series consists of five novel pteridines (2a-e, Figure 6) modified to improve interactions with PTR1 and parasite DHFR and to exploit the differences in pocket sizes and residues between the parasitic targets and the hDHFR off-target.

\section{Rationale for PABA modifications}

As a first modification to the PABA moiety, in $\mathbf{3 a}$, we replaced the PABA phenyl group with benzyl (Figure 6). The additional hydrophobic spacer can interact with hydrophobic target residues, while resulting in a shifted position of the hydrophilic linker amide. The positioning of hydrophobic and hydrophilic residues surrounding the PABA moiety and the amide linker in the human off-target is different from the parasite targets PTR1 and DHFR, which can be exploited to improve selectivity. For the same reason, the amide linker position was also shifted in $\mathbf{3} \mathbf{b}$ by substituting the PABA moiety with meta-aminobenzoic acid. 

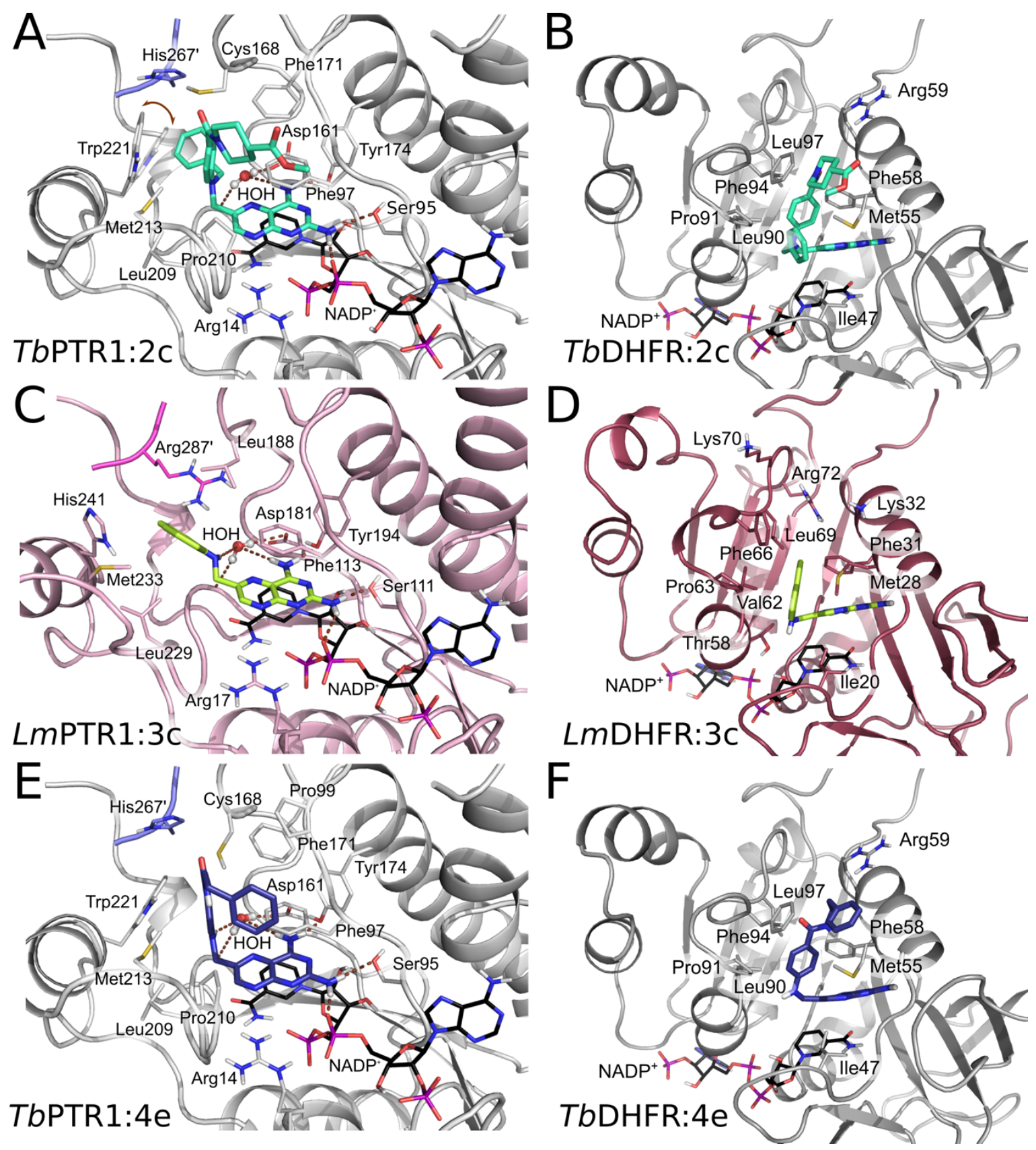

Figure 5. Views of the binding sites showing docked poses of selected pteridine-based inhibitors in the target proteins: TbPTR1 (pale gray) (A,E), TbDHFR (dark gray) (B,F), LmPTR1 (pale pink) (C) and LmDHFR (dark pink) (D). (A) An induced fit (IF) MTX-like docking pose for compound 2c (cyan carbons) in TbPTR1 in the presence of a conserved water molecule (ball-and-stick representation): Trp221 moves (indicated by a brown arrow) to make room for the phenyl of $2 c$. (B-F) Rigid-body docking poses of 2c in TbDHFR (B), 3c (lime carbons) in $L m P T R 1$ and $L m D H F R(C, D)$ and $4 \mathbf{e}$ (purple carbons) in TbPTR1 and TbDHFR (E,F); see text for discussion. Docked poses are shown for N1-deprotonated compounds, but similar orientations were observed for the N1-protonated forms (see Figure S6). For PTR1, all docking poses shown were obtained in the presence of conserved structural water molecules. Generally, similar poses were observed for docking without water. In all panels, proteins are shown in cartoon representation with the important interacting residues (compare Figure 4A) and the NADPH/NADP ${ }^{+}$cofactor shown in sticks (carbons colored according to protein and black, respectively). Residues His267' and Arg287' from the neighboring subunit are shown in lavender and magenta in TbPTR1 and LmPTR1, respectively. Hydrogen bonds are represented by brown dashed lines. Further IF docking poses are shown in Figures $\mathbf{S 7}$ and $\mathbf{S 8}$. 
A second key feature of the targets vs. off-targets that was used to inform the design of the PABA-series relates to the compound tail: Tail regions are solvent-exposed in PTR1 and thus have poorly defined interactions (Figure 4B). In contrast, in DHFR, the tail region is enclosed, and strong interactions occur with the hDHFR off-target. ${ }^{31}$ We therefore shortened the tail region to achieve full enclosure in the PTR 1 binding pocket by replacing PABA by naphthalene (3c) or benzene moieties (non-substituted, $\mathbf{3 d}$; or substituted with $-\mathrm{CF}_{3}, \mathbf{3 e}$ ). Docking results showed that the smaller tail fully resides in the PTR1 binding pocket (Figure 5C) and is stabilized by surrounding hydrophobic residues, not only in PTR1, but also in parasite DHFR (Figure 5CD). Rigid-body docking studies suggested that the bulky naphthalene of $3 \mathrm{c}$ may be particularly beneficial in $L m P T R 1$, since this target has a more elongated, open pocket compared to TbPTR1 (Figure 4C). The PABA moiety modifications are therefore suitable for modulating the compound interaction profile in a species-specific manner.

In summary, the PABA series contains 5 new pteridine derivatives (3a-3e, Figure 6) designed to improve selectivity by exploiting the different surroundings of bound PABA moieties in hDHFR in comparison to the parasite target proteins.

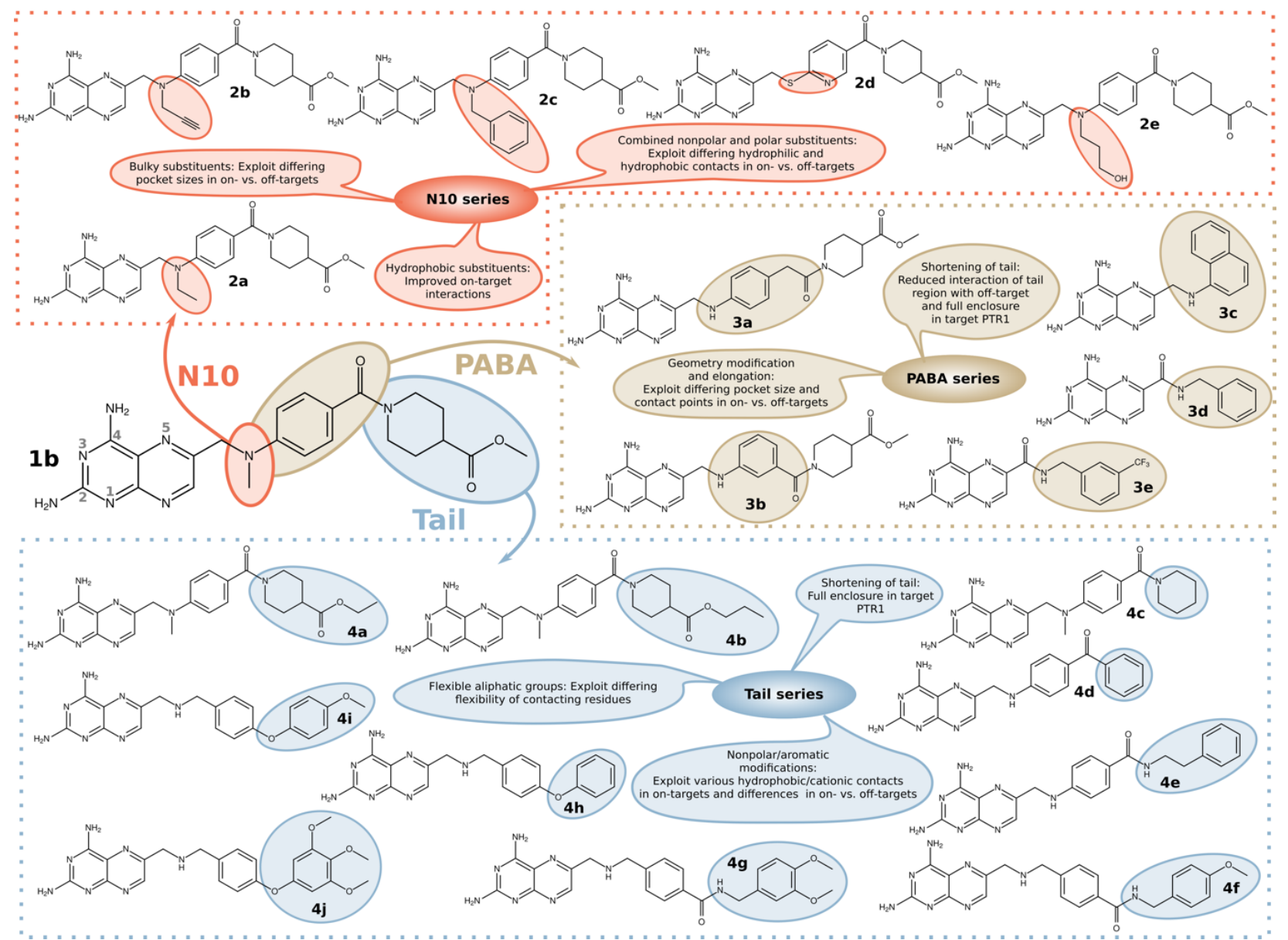

Figure 6. Overview of the modifications in the N10, PABA and Tail modules explored in the designed compound series with respect to the reference compound 1b. Synthesized members of each designed series are shown in the framed boxes along with the key objectives addressed with the respective modifications. See text for details. 


\section{Rationale for Tail modifications}

The surrounding of the compound tail features several hydrophobic residues, particularly in the two T. brucei targets (Figure 4A). Directional interactions with the tail moiety may have limited benefit for the binding affinity in PTR1, since the flexibility of the solvent-exposed tail likely has an entropic contribution. Hydrophobic interactions are geometrically less restrained than, for instance, hydrogen bonds. Therefore, anticipating less pronounced entropic penalties on binding in our designed derivatives, we replaced the methyl ester in the tail of $\mathbf{1 b}$ by the more flexible ethyl and propyl in $\mathbf{4 a}$ and $\mathbf{4 b}$, respectively. Two aspects may result in selectivity benefits from this approach: The tail region is enclosed by more hydrophobic moieties in parasite DHFR than in the hDHFR off-target (Figure 4A) and residues surrounding the tail have previously been demonstrated to show differing conformational variability in the crystal structures when comparing parasitic targets with the off-target. ${ }^{31}$

To combine exploitation of the differing pattern of hydrophobic residues in the tail environment of targets and off-targets with improved enclosure in PTR1 (Figure 4AB), we further modified the tail to an unsubstituted piperidine $(\mathbf{4 c}$ ) or replaced piperidine with an unsubstituted benzene (4d). Compound $4 \mathrm{e}$, with benzene attached via a flexible ethyl linkage to an MTXlike amide, can benefit from nonpolar and aromatic residues surrounding the tail in PTR1 and parasite DHFR and, according to docking predictions, readily adapt to their differing placement in the on-targets, see Figure 5EF. The docking studies additionally suggest that the flexible aromatic tail can form cation- $\pi$ interactions with positively charged residues in the entrance of the DHFR pocket (e.g. Arg59 of TbDHFR, Figures 4A, 5F). Additional hydrophobic residues in the target pocket entrance regions, like Pro99 of TbPTR1 (Figure 4A), can be targeted with an altered geometry in combination with methoxylations: $\mathbf{4 f}$ and $\mathbf{4 g}$ combine a one-carbon spacer between N10 and PABA and amide-linked methoxylated tail portions. In addition, an etheryl linkage to a non-substituted (4h), methoxylated (4i) or trimethoxylated (4j) benzyl group was explored. Compounds $\mathbf{4 f - 4} \mathbf{j}$ were collectively designed to interact with the different hydrophobic, aromatic and positively charged surrounding residues found around the tail region in the various targets (Figure 4A).

Taken together, the tail series comprises 10 new pteridines (4a-4j, Figure $\mathbf{6})$ with modified tails to target residue patterns distinguishing on- and off-targets and the distinct surroundings of the tail in PTR1 vs. DHFR.

\section{Synthesis of pteridine derivatives with high yield}

26 new 2,4-diaminopteridine derivatives and the reference compounds $\mathbf{1 b}$ and 1c were (re)synthesized as reported in Schemes 1-8. We applied our methodology for improved reaction yield of the chemical pteroid step to provide a key intermediate for most of the designed compounds. ${ }^{33}$ Displacement of the chloride of 6-(chloromethyl)pteridine-2,4-diamine 
hydrochloride $(29$, Scheme 1$)$ by the appropriate substituted anilines and aliphatic aminoderivatives was carried out in $\mathrm{N}, \mathrm{N}$-dimethylacetamide (DMA) at $60^{\circ} \mathrm{C}$ microwave (MW) to provide 1b-c, 2a-e, 3a-c, 4a-j, 5a-f in high yields of $70-90 \%$ with reduced reaction time (Schemes 2-7). ${ }^{33}$<smiles>Nc1nc(N)c2nc(CO)cnc2n1</smiles>

$\mathrm{Pt}-\mathrm{OH}$<smiles>CC(C)CCl</smiles>

29<smiles>Nc1nc(N)c2nc(CN(P)P)cnc2n1</smiles>

1b-c, 2a-c, 2e, 3a-c, 4a-j, 5a-f

$\mathrm{R}=\mathrm{R}^{1}=$ aliphatic or aromatic amines moiety

2d $R=R^{1}=$ pyridine-2-thiol moiety

Scheme 1. Synthesis of derivatives of compound 29. Reagents and conditions: (i) $\mathrm{SOCl}_{2}$, reflux, $12 \mathrm{~h}$. $70 \%$ yield; (ii) 29 (1.2 equiv.), corresponding amine derivative (1 equiv.), $\mathrm{K}_{2} \mathrm{CO}_{3}$ (3 equiv.), $\mathrm{KI}$ (0.1 equiv.), DMA, 60 ${ }^{\circ}, 20^{\prime}-$ 30 ' MW.

The PABA amine functionalization was achieved by selective alkylation of primary amines to secondary amines using nitriles as an alkylating reagent with $\mathrm{Pd} / \mathrm{C}$ for intermediates 32-33. ${ }^{34,35}$ Conventional alkylation of the latter with propargyl bromide or (bromomethyl)benzene resulted in derivatives 34 and 35, respectively (Scheme 2).<smiles>Nc1ccc(C(=O)O)cc1</smiles>

$30-35$<smiles>CC1CCNCC1</smiles><smiles>[R]C1CCNCC1</smiles>

36- 39

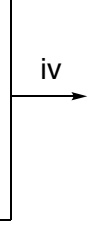<smiles>CCOCCC1CCN(C(=O)c2ccc(N)cc2)CC1</smiles><smiles>Nc1nc(N)c2nc(CCl)cnc2n1</smiles>

29

1b-c, 2a-c, 2e, 4a-c, 5c

\begin{tabular}{|c|c|c|c|c|c|c|}
\hline & $R=$ & $\mathrm{R}^{1}=$ & & & $R=$ & $\mathrm{R}^{1}=$ \\
\hline 30 & $\mathrm{CH}_{3}$ & - & 40 & 1b & $\mathrm{CH}_{3}$ & $\mathrm{COOCH}_{3}$ \\
\hline 31 & $\mathrm{H}$ & _- & 41 & 1c & $\mathrm{H}$ & $\mathrm{COOCH}_{3}$ \\
\hline 32 & $\mathrm{CH}_{2} \mathrm{CH}_{3}$ & _ & 42 & $2 a$ & $\mathrm{CH}_{2} \mathrm{CH}_{3}$ & $\mathrm{COOCH}_{3}$ \\
\hline 33 & $\mathrm{CH}_{2} \mathrm{CH}_{2} \mathrm{CH}_{2} \mathrm{OH}$ & _ & 43 & $2 e$ & $\mathrm{CH}_{2} \mathrm{CH}_{2} \mathrm{CH}_{2} \mathrm{OH}$ & $\mathrm{COOCH}_{3}$ \\
\hline 34 & $\mathrm{CH}_{2} \mathrm{CCH}$ & _ & 44 & $2 b$ & $\mathrm{CH}_{2} \mathrm{CCH}$ & $\mathrm{COOCH}_{3}$ \\
\hline 35 & $\mathrm{CH}_{2} \mathrm{C}_{6} \mathrm{H}_{5}$ & _- & 45 & $4 b$ & $\mathrm{CH}_{3}$ & $\mathrm{COOCH}_{2} \mathrm{CH}_{2} \mathrm{CH}_{3}$ \\
\hline 36 & - & $\mathrm{COOCH}_{3}$ & 46 & $4 a$ & $\mathrm{CH}_{3}$ & $\mathrm{COOCH}_{2} \mathrm{CH}_{3}$ \\
\hline 37 & - & $\mathrm{COOCH}_{2} \mathrm{CH}_{2} \mathrm{CH}_{3}$ & 47 & $5 c$ & $\mathrm{CH}_{2} \mathrm{CH}_{3}$ & $\mathrm{COOCH}_{2} \mathrm{CH}_{3}$ \\
\hline 38 & _ & $\mathrm{COOCH}_{2} \mathrm{CH}_{3}$ & 48 & 2c & $\mathrm{CH}_{2} \mathrm{C}_{6} \mathrm{H}_{5}$ & $\mathrm{COOCH}_{3}$ \\
\hline 39 & _ & $\mathrm{H}$ & 49 & $4 c$ & $\mathrm{CH}_{3}$ & $\mathrm{H}$ \\
\hline
\end{tabular}

Scheme 2. Synthesis of compounds $1 \mathrm{~b}-\mathrm{c}, 2 \mathrm{a}-\mathrm{c}, 2 \mathrm{e}, 4 \mathrm{a}-\mathrm{c}, 5 \mathrm{c}$ and intermediates $32-35,37,38,40-49$. Reagents and conditions: compounds 30,31, 36, 39 were purchased from Sigma; (i) acetonitrile or 3- 
hydroxypropanenitrile, 10\% $\mathrm{Pd} / \mathrm{C}, \mathrm{NH}_{4} \mathrm{OAc}$ (1 equiv.) $\mathrm{CH}_{3} \mathrm{OH}, \mathrm{H}_{2}, \mathrm{rt}, 24-36 \mathrm{~h}$ (32-33); (ii) alkyl halide (propargyl bromide, (bromomethyl)benzene) (0.5 equiv.), $\mathrm{K}_{2} \mathrm{CO}_{3}$ (2 equiv.), DMF dry, rt, 24h (34-35); (iii) $\mathrm{SOCl}_{2}$ (4 equiv.), propanol (for 37), $\mathrm{EtOH}$ (for 38), reflux, $7-12 \mathrm{~h}$ (89 and $96 \%$ yield); (iv) $\mathrm{EDC} \cdot \mathrm{HCl}(1.1$ equiv.), HOBt (0.1 equiv.), TEA (2-3 equiv.), DMF, rt, overnight (40-49); (v) 29 (1.2 equiv.), corresponding amine derivative (1 equiv.), $\mathrm{K}_{2} \mathrm{CO}_{3}$ (3 equiv.), $\mathrm{KI}(0.1$ equiv.), DMA, 20' MW (1b-c, 2a-c, 2e, 4a-c, 5c).

The reductive alkylation of amines using nitriles was also used to obtain $\mathbf{5 1}$ and $\mathbf{7 4}$ in Schemes 3 and 7 . The isonipecotic acid derivatization was achieved via Fischer esterification using the reagent-solvents propanol (37) and EtOH (38), respectively; methyl isonipecotate (36) and piperidine (39) were purchased from Sigma (Scheme 2). The intermediate acid derivatives 30-35, d-e were condensed to amides through a coupling reaction with the respective amines $36-39$ and $\mathbf{g}$ using $\mathrm{EDC} \cdot \mathrm{HCl}$ in dimethylformamide (DMF) as the coupling agent to provide the intermediate products 40-49, 71-72 and 75, which were then made to react with 29 to obtain the final compounds (1b-c, 2a-d, 3b, 4a-c, 4e, 5c; Schemes 2, 6, 7).

Using the same method, we synthesized the elongated compounds 3a, 4f-g, 5a-b, characterized by a carbon spacer in the PABA moiety (Schemes 3-4).

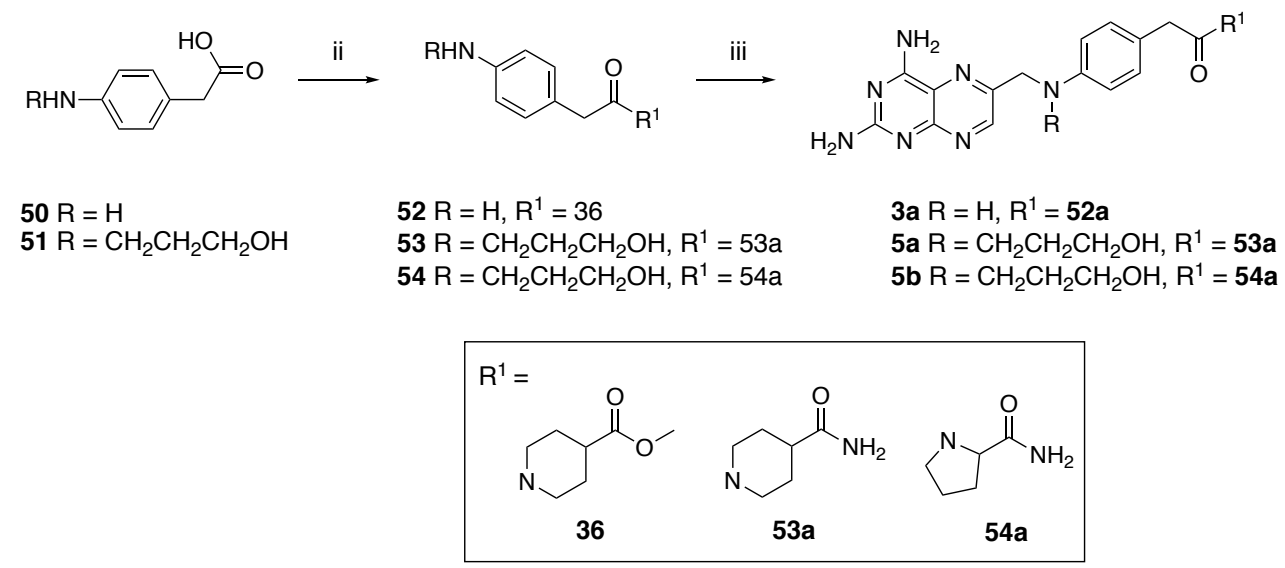

Scheme 3. Synthesis of compounds 3a, 5a-b. Reagents and conditions: (i) 3-hydroxypropanenitrile, 10\% Pd/C, $\mathrm{NH}_{4} \mathrm{OAc}$ (1 equiv.), $\mathrm{CH}_{3} \mathrm{OH}, \mathrm{H}_{2}$, rt, $24 \mathrm{~h}$ (51); (ii) $\mathrm{EDC} \cdot \mathrm{HCl}$ (1.1 equiv.), $\mathrm{HOBt}(0.1$ equiv.), TEA (2-3 equiv.), DMF, rt, overnight (52-54); (iii) 29 (1.2 equiv.), corresponding amine derivative (1 equiv.), $\mathrm{K}_{2} \mathrm{CO}_{3}$ (3 equiv.), $\mathrm{KI}$ ( 0.1 equiv.), DMA, 20' MW (3a, 5a-b).

To obtain 4f-g (Scheme 4), an additional protection step reaction to guide selective amide functionalization was necessary. The selectivity was achieved via Boc-protection in the first step of the reaction of $\mathbf{b}$ to obtain 55 , which was then coupled with the respective aliphatic amine to give 56-57. The target amines were finally obtained by a deprotection step carried out in $30-40 \%$ trifluoroacetic acid/dichloromethane (TFA/DCM) in quantitative yield.

The phenoxyphenyl-methanamine derivative intermediates (Scheme 5) were synthesized starting from 4-fluorobenzaldehyde and the respective phenol derivates 60-62 by an $S_{N} A r$ reaction. Subsequently, the primary amines $66-68^{36}$, or functionalized 
amines 69-70 (obtained via a one-pot reductive step), were reacted with 29 to obtain 4h-j, 5e-f.<smiles>[R]c1cc(CNC(=O)c2ccc(CN)cc2)ccc1OC</smiles><smiles></smiles>

Scheme 4. Synthesis of compounds 4f-g. Reagents and conditions: (i) di-tert-butyl pyrocarbonate (1.05 equiv), dioxane $\mathrm{H} 2 \mathrm{O} \backslash 1 \mathrm{~N} \mathrm{NaOH} 1 \backslash 1 \backslash 1 \mathrm{VVVV}$, rt, $6 \mathrm{~h}$ (55) (i) EDC·HCl (1.1 equiv.), HOBt (0.1 equiv), TEA (2-3 equiv.), DMF, rt, overnight (56-57); (iii) TFA, DCM, rt; (58-59); (iv) 29 (1.2 equiv.), corresponding amine derivative (1 equiv.), $\mathrm{K}_{2} \mathrm{CO}_{3}$ (3 equiv), $\mathrm{KI}$ (0.1 equiv.), DMA, 20' MW (4f-g).

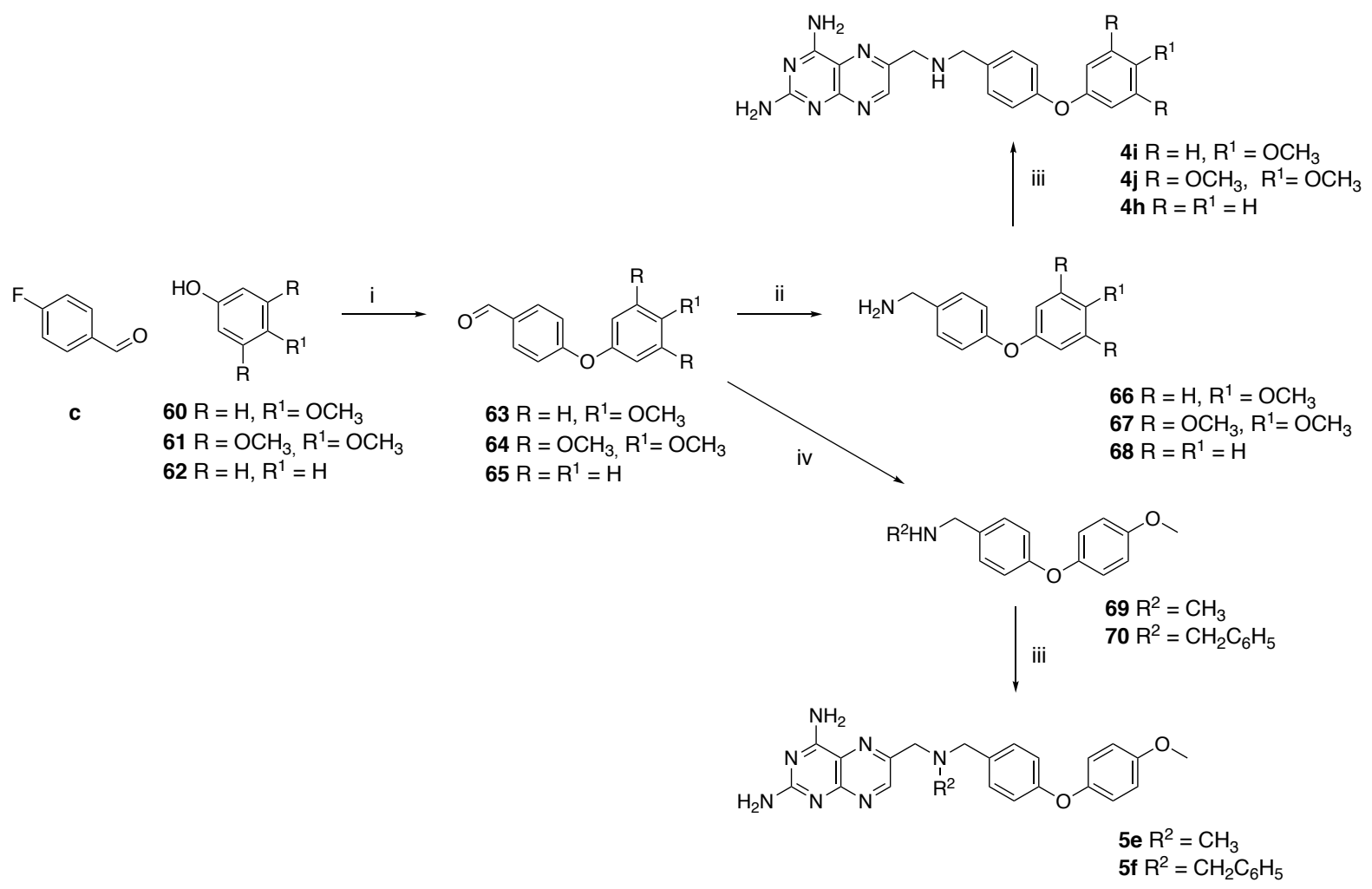

Scheme 5. Synthesis of compounds 4h-j, 5e-f. Reagents and conditions: (i) $\mathrm{K}_{2} \mathrm{CO}_{3}$ (3 equiv.), DMF, reflux, 16-18 h (63-65); (ii) $\mathrm{NH}_{2} \mathrm{OH} . \mathrm{HCl}$ (1.2 equiv.), $\mathrm{EtOH}, \mathrm{rt},>1 \mathrm{~h}$ followed by $\mathrm{Zn}$ dust (2.5 equiv.) in $12 \mathrm{M} \mathrm{HCl}(4$ equiv.), rt, 15'(66-68), (iii) 29 (1.2 equiv.), corresponding amine derivative (1 equiv.) $\mathrm{K}_{2} \mathrm{CO}_{3}$ (3 equiv.), $\mathrm{KI}$ (0.1 equiv.), DMA, $20^{\prime} \mathrm{MW}$ (4h-j, 5e-f); (iv) methylamine (for 69) or benzylamine (for 70) EtOH dry, 60 $\mathrm{C}, 3 \mathrm{~h}$, then $\mathrm{NaBH}_{4}(1.5$ equiv.), rt, $2 \mathrm{~h}$.

Compounds $\mathbf{3 c}, \mathbf{4} \mathbf{d}$ and $\mathbf{5 d}$, with a higher steric hindrance, were obtained with slightly increased reaction time in a good yield. Finally, to obtain $\mathbf{3 d - e}$, it was necessary to first perform an oxidation reaction. Treatment of $\mathrm{Pt}-\mathrm{OH}$ in acetone/0.5 M phosphate buffer 
at $\mathrm{pH} 7(1: 1 \mathrm{vlv})$ with $\mathrm{KMnO}_{4}$ gave the oxidized analogue $\mathbf{7 6}$, which was subsequently coupled with the selected aliphatic amine to obtain the desired amides (Scheme 8).<smiles>COC(=O)C1CCN(C(=O)c2cccc(N)c2)CC1</smiles>

d<smiles>COC(=O)C1CCN(C(=O)c2cccc(NCc3cnc4nc(N)nc(N)c4n3)c2)CC1</smiles>

3b

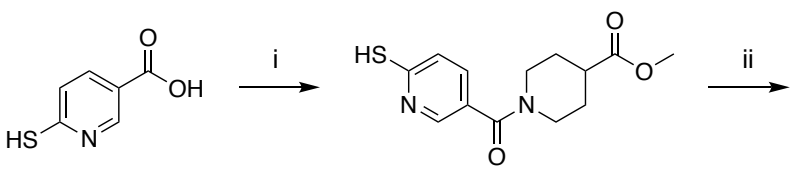

72<smiles>COC(=O)C1CCN(C(=O)c2ccc(SCc3cnc4nc(N)nc(N)c4n3)nc2)CC1</smiles>

2d

Scheme 6. Synthesis of compounds $\mathbf{3 b}$ and $\mathbf{2 d}$. Reagents and conditions: (i) EDC $\cdot \mathrm{HCl}(1.1$ equiv.), $\mathrm{HOBt}(0.1$ equiv.), TEA (2-3 equiv.), DMF, rt, overnight (71-72); (ii) 29 (1.2 equiv.), corresponding amine derivative (1 equiv.), $\mathrm{K}_{2} \mathrm{CO}_{3}$ (3 equiv.), $\mathrm{KI}(0.1$ equiv.), DMA, 20' MW (3b, $2 \mathrm{~d})$.<smiles>Nc1ccc(C(=O)c2ccccc2)cc1</smiles>

$\begin{aligned} \text { ii } \longrightarrow 73 \mathrm{R} & =\mathrm{H} \\ \longrightarrow 74 \mathrm{R} & =\mathrm{CH}_{2} \mathrm{CH}_{3}\end{aligned}$<smiles>Nc1cccc2ccccc12</smiles>

$f$<smiles></smiles>

$4 d \mathrm{R}=\mathrm{H}$ $\mathbf{5 d ~ R}=\mathrm{CH}_{2} \mathrm{CH}_{3}$<smiles>Nc1nc(N)c2nc(CNc3cccc4ccccc34)cnc2n1</smiles>

3c<smiles>CC#CC(=O)c1ccc(N)cc1</smiles>

31 g
75<smiles>Nc1nc(N)c2nc(CNc3ccc(C(=O)NCCc4ccccc4)cc3)cnc2n1</smiles>

Scheme 7. Synthesis of compounds 3c, 4d-e, and 5d. Reagents and conditions: (i) 29 (1.2 equiv.), corresponding amine derivative (1 equiv.), $\mathrm{K}_{2} \mathrm{CO}_{3}$ (3 equiv.), $\mathrm{KI}(0.1$ equiv.), DMA, 30' MW (3c, 4d-e, 5d). (ii) acetonitrile, $10 \% \mathrm{Pd} / \mathrm{C}, \mathrm{NH}_{4} \mathrm{OAc}$ (1 equiv.) $\mathrm{CH}_{3} \mathrm{OH}, \mathrm{H}_{2}$, rt, 24-36 h (74); (iii) $\mathrm{EDC} \cdot \mathrm{HCl}$ (1.1 equiv.), $\mathrm{HOBt}(0.1$ equiv.), TEA (2-3 equiv.), DMF, rt, overnight (75).<smiles>Nc1nc(N)c2nc(CO)cnc2n1</smiles>

Pt-OH<smiles>CC(C)C</smiles>

76<smiles>Nc1nc(N)c2nc(C(=O)NCc3cccc(P)c3)cnc2n1</smiles>

$$
\text { 3d } \mathrm{R}=\mathrm{H}
$$$$
\text { 3e } \mathrm{R}=\mathrm{CF}_{3}
$$

Scheme 8. Synthesis of compounds 3d-e. Reagents and conditions: (i) $\mathrm{KMnO}_{4}$, acetone/0.5 M phosphate buffer at $\mathrm{pH} 7$ (1:1 VV); (ii) EDC. $\mathrm{HCl}$ (1.1 equiv.), HOBt (0.1 equiv.), TEA (2-3 equiv.), DMF, rt, overnight. 


\section{Crystal structures for the PTR1 targets confirm the predicted interactions and that the}

pteridine derivatives adopt a methotrexate inhibitor-like orientation

The structures of TbPTR1 with two new pteridines, $\mathbf{2} \mathbf{a}$ and $\mathbf{2 e}$, and that of $L m P T R 1$ with $\mathbf{2 e}$, were determined to $1.20 \AA, 1.11 \AA$ and $2.10 \AA$ resolution, respectively (see Tables S2-S3). The structures contain functional enzyme tetramers in the crystallographic asymmetric unit with a similar structure to those previously determined ${ }^{37,38}$. In all complexes, the compounds adopt MTX-like binding modes (Figure 7AB).

In line with the docking predictions, the overall structure of the TbPTR1 complexes resembles the complex with $\mathbf{1 b}$ (compare Figure $\mathbf{7 A}$ with $\mathbf{3 C}$ ). In agreement with the design objective, the $\mathrm{N}$-ethyl moiety of $\mathbf{2 a}$ was found to form van der Waals interactions with Val206 and Trp221 on the hydrophobic side of the pocket (Figure 7A). The bulkier N-propylhydroxyl moiety of $\mathbf{2 e}$ forms direct and water-mediated hydrogen bonds with Asp161 and receives an intramolecular hydrogen bond from the amine in position 4 on the pteridine system (Figure S2C). The structure of LmPTR1 in complex with $2 \mathbf{e}$ (Figure 7B) closely resembles that observed in TbPTR1, except for the terminal piperidine moiety (Figure S2CD). The latter moiety is highly flexible - a possible orientation is reported in the crystal structure, but further orientations cannot be excluded.

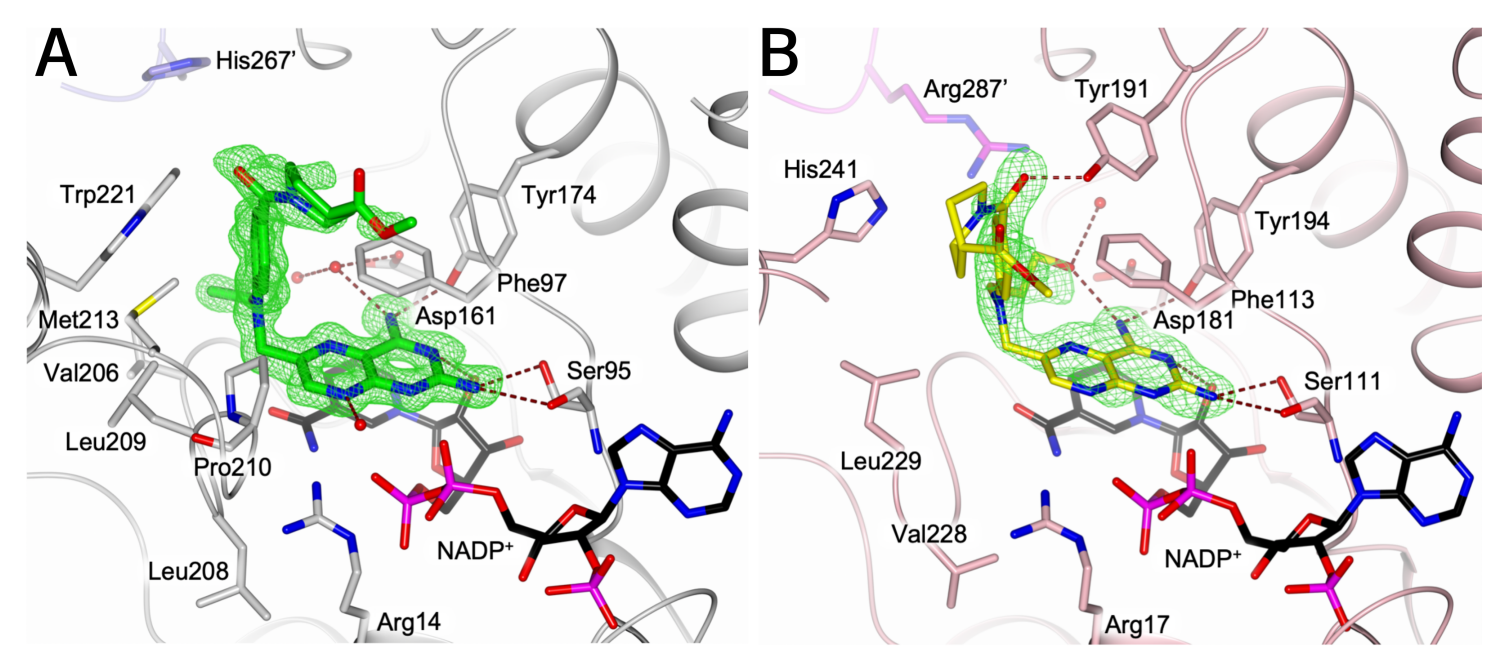

Figure 7. Views of the binding sites of crystal structures of complexes of pteridine-based inhibitors in TbPTR1 and LmPTR1 determined in this work, which confirm the predicted MTX-like binding modes. (A) 2a (green carbons) in TbPTR1 (gray cartoon, His267' from the neighboring subunit in lavender) and (B) $2 \mathbf{e}$ (yellow carbons) in LmPTR1 (pink cartoon, Arg287' from the neighboring subunit in magenta). Water molecules are shown as red spheres and the inhibitors are surrounded by the omit map (green wire) contoured at the $2.5 \sigma$ level. Interacting residues and the NADPH/NADP ${ }^{+}$cofactor are shown in sticks (carbons colored according to protein and black, respectively). Hydrogen bonds are represented by brown dashed lines.

\section{Designed pteridine derivatives have improved target and off-target enzyme inhibitory activities}

The measured inhibitory activities of compounds $2 \mathrm{a}-\mathbf{e}, 3 \mathbf{a}-\mathbf{e}$ and $4 \mathrm{a}-\mathrm{j}$ against the targets TbPTR1, TbDHFR, LmPTR1, LmDHFR, and the off-targets hDHFR and hTS, are given in 
Figure 8 and Table S1. Overall, the inhibitory activities against the PTR1 targets for the designed compounds are improved, as are PTR1 vs. off-target selectivities.

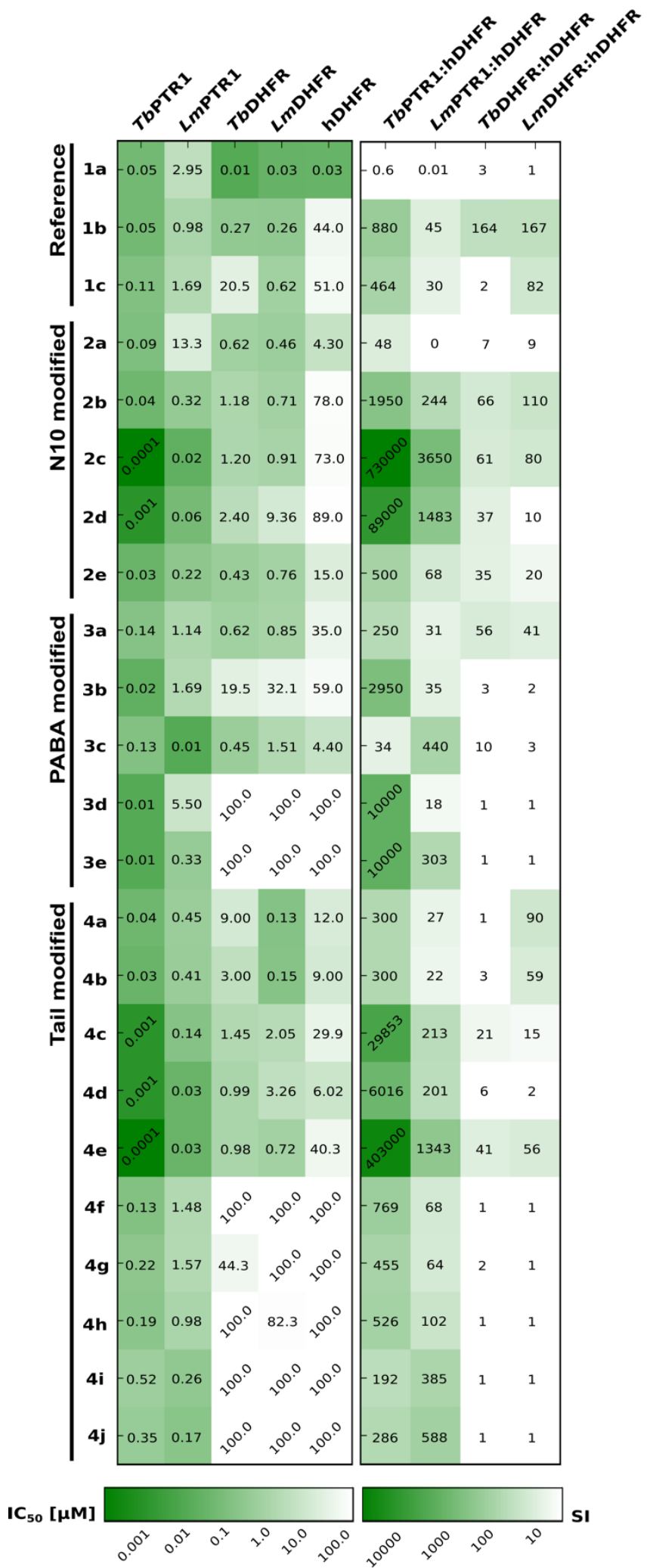

Figure 8. Inhibitory activities ( $\mathrm{IC}_{50}$ values, left) and selectivities (Selectivity indices (SI), right) of compounds of the designed N10-, PABA-, and Tail-modified series and selected reference compounds against the targets TbPTR1, LmPTR1, TbDHFR, LmDHFR and the off-target hDHFR. All values, as well as data for hTS, are reported in Table S1. Greener boxes show higher inhibition and selectivity. 
N10 modifications yield improved PTR1 inhibitors with similar selectivity trends for parasite DHFRs. The N10-modified compounds (2a-e; Figure 6) are improved PTR1 inhibitors in comparison to 1b, except for 2a (1b IC 50 TbPTR1 50 nM, LmPTR1 $1 \mu \mathrm{M}$; N10 series $\mathrm{IC}_{50}$ TbPTR1 <0.1 - $90 \mathrm{nM}$; LmPTR1 0.02-13.3 $\mu \mathrm{M}$; Figure 8). 2c is the best in the series with a picomolar $\mathrm{IC}_{50}$ against $T b P T R 1$ and an $\mathrm{IC}_{50}$ of $20 \mathrm{nM}$ against $L m P T R 1$.

All compounds are roughly similar to $\mathbf{1 b}$ in parasite DHFR inhibition (1b IC 50 TbDHFR and LmDHFR 0.3 $\mu \mathrm{M}$; N10 series IC 50 TbDHFR 0.4-2.4 $\mathrm{M}$, LmDHFR 0.5-9.4 $\mu \mathrm{M}$ ), and selectivity over hDHFR ranges from 7- to 66-fold for TbDHFR and 9- to 110-fold for LmDHFR, which is somewhat lower than for $\mathbf{1 b}$ (SI TbDHFR/hDHFR = 164 and $L m D H F R / h D H F R=167)$. Thus, mainly PTR1 inhibition benefits from the selected N10 modifications.

PABA modifications lead to strong variations in the target inhibition profile. The modifications of the PABA moiety in the PABA series (compounds 3a-e; Figure 6) distinctly affect the inhibitory activities against the targets. In particular, smaller compounds with wellenclosed binding poses show improvements in inhibitory activity that vary for different PTR1 variants: $3 c$, in contrast to most of the studied pteridines, is 13-fold more potent towards LmPTR1 than TbPTR1 ( $\mathrm{IC}_{50} 10 \mathrm{nM}$ and $130 \mathrm{nM}$, respectively), in line with its predicted steric fit to the LmPTR1 binding pocket shape (compare Figures $4 \mathrm{C}$ and $5 \mathrm{~B}$ ). Full enclosure and stabilizing interactions with hydrophobic residues lining the pocket entrance likewise probably contribute to an around 10-fold higher potency of $\mathbf{3 d}$ and $\mathbf{3 e}$ against TbPTR1 than the most similar reference compound 1c (lacking an N10 substitution) ( $\mathrm{IC}_{50} \mathbf{3 d}, \mathbf{3 e :} 10 \mathrm{nM}$; $1 \mathrm{c}: 110 \mathrm{nM}$ ). Whereas $\mathbf{3 d}$ and $\mathbf{3 e}$ do not show inhibitory activity against the parasite DHFR targets, $\mathbf{3 c}$ shows similar activity against $L m D H F R$ to $1 \mathrm{c}\left(\mathrm{IC}_{50} 1.5\right.$ and $0.6 \mu \mathrm{M}$, respectively) and displays higher activities against both TbDHFR (IC 50 1c: $20.5 \mu \mathrm{M}$; 3c: $0.5 \mu \mathrm{M})$ and hDHFR (IC 50 1c: 51 $\mu \mathrm{M} ; 3 \mathrm{c}: 4 \mu \mathrm{M})$. A one-carbon spacer to shift the position of the PABA carbonyl in $\mathbf{3 a}$ with respect to 1c improves inhibition of TbDHFR $\left(\mathrm{IC}_{50} 0.6 \mu \mathrm{M}\right)$ while not significantly affecting inhibition of $L m D H F R$ and hDHFR. Thus, $3 a$ is more selective against TbDHFR than the reference 1c (SI: 56 vs. 2).

Taken together, alterations to the PABA moiety, due to its central location in the compound scaffold, different pocket sizes and surrounding residue patterns in targets (Figure 4A), display highly variable effects on the activity profiles.

Alterations in tail geometry boost PTR1 inhibition but can reduce DHFR inhibition. In the Tail-modified series (compounds 4a-j; Figure 6), hydrophobic and aromatic residues lining the pocket entrance region of PTR1 were exploited by either tail elongation or shortening. The interactions of these residues with the flexible aromatic tail of $4 \mathrm{e}$ (see Figure $5 \mathrm{E}$ ) likely contribute to the boost of the $\mathrm{IC}_{50}$ against TbPTR1 to the picomolar range and to $30 \mathrm{nM}$ against 
LmPTR1 - >1000-fold and 57-fold improvements, respectively, in PTR1-inhibitory potencies compared to reference compound 1c. The shortened tails of $4 \mathrm{c}$ (unsubstituted piperidine) and $\mathbf{4 d}$ (benzene) are stabilized by the same residues and likely benefit from a better enclosure in the PTR1 pocket. Both compounds show improved TbPTR1 and LmPTR1 inhibition compared to $1 \mathbf{b}\left(\mathrm{IC}_{50}\right.$ TbPTR1 4c, 4d: $1 \mathrm{nM}$ vs. 1b: $50 \mathrm{nM}$; LmPTR1 4c: $0.1 \mu \mathrm{M}, 4 \mathrm{~d}: 0.03 \mu \mathrm{M}$ vs. $1 \mathrm{~b}: 1.0$ $\mu \mathrm{M})$.

However, shortening of the tail diminishes the inhibition of parasite DHFR, whereas it either does not affect or increases inhibition of the off-target hDHFR. Revisiting the docking predictions provides a possible explanation for this: The piperidine/benzene groups in the tails of $\mathbf{4 c}$ and $\mathbf{4 d}$ can form more extended hydrophobic interactions with Phe31 of hDHFR than with the corresponding methionine in the parasite DHFR variants (Figure 9). In the parasite protein, moreover, Asn64 in the pocket entrance of hDHFR is replaced by phenylalanine, which, upon interaction with the compound tail, becomes solvent-exposed.
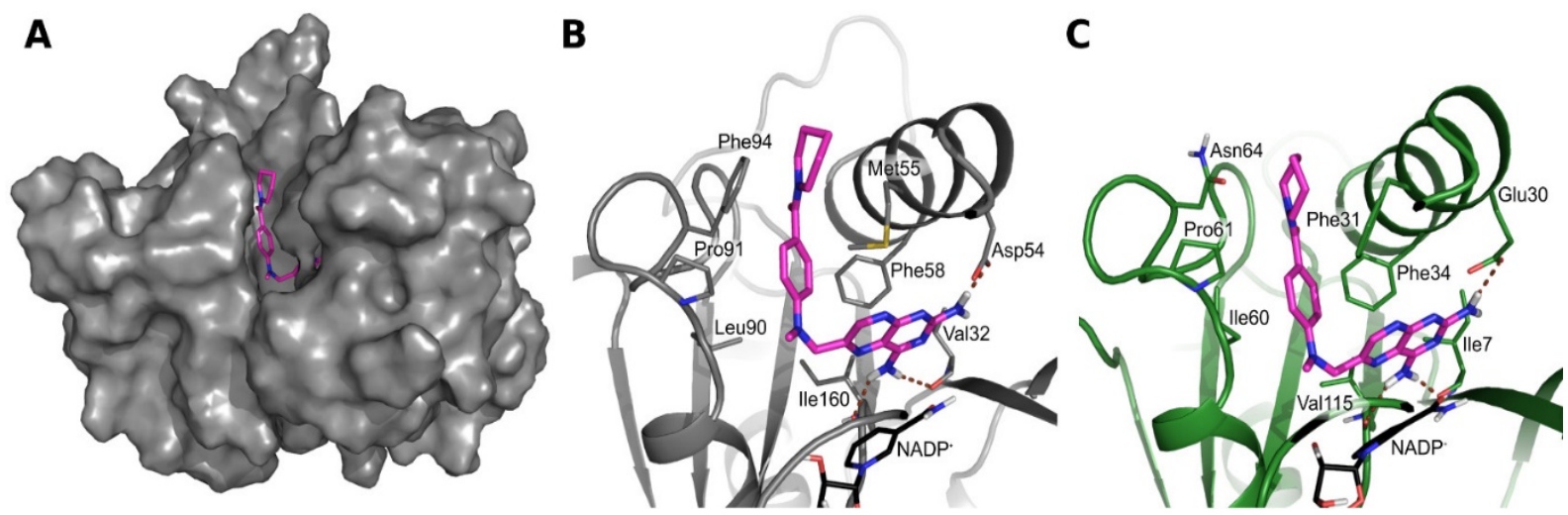

Figure 9. Docking poses for compound $4 \mathrm{c}$ from the Tail series (magenta carbons) in (A, B) TbDHFR and (C) hDHFR, showing differences in exposure and interactions of the PABA and Tail moieties in the two DHFRs. (A) The TbDHFR pocket accommodates $\mathbf{4 c}$ with its tail enclosed by surrounding residues. hDHFR has a similar shape. TbDHFR is shown in gray surface representation. $(B, C)$ Views of the binding sites of TbDHFR and hDHFR, which are shown in cartoon representation in gray and green, respectively. Important interacting residues and the NADPH/NADP ${ }^{+}$cofactor (black carbons) are shown as sticks. Hydrogen bonds are indicated by brown dotted lines. While the orientation of $\mathbf{4 c}$ is rather similar in both DHFR variants, the tail moiety is more solvent-exposed in TbDHFR: The PABA benzene and piperidine of 4c compete for interactions with Phe94 of TbDHFR, which thereby becomes exposed to the solvent. In hDHFR, the corresponding exposed residue is the polar Asn64 and the tail of 4c can interact with Phe31 deeper in the pocket, rendering the mode of binding more favorable in hDHFR. The results are presented for N1-deprotonated compounds, but similar observations were made with N1 protonated (Figure S6).

Pocket size and interaction pattern differences between LmDHFR and other DHFR variants, as also discussed for the PABA series, also affect the Tail-modified compounds: For instance, 4d is more active against both TbDHFR and hDHFR than 1c $\left(I_{50}\right.$ TbDHFR 1 vs. $21 \mu \mathrm{M}$, hDHFR 6 vs. $51 \mu \mathrm{M})$, while both compounds show similar activity for $L m D H F R$.

Summary of the compound activity profiles for the N10, PABA and Tail-modified series.

Taken together, most of the new pteridine derivatives display 1-2-fold greater inhibition of TbPTR1 than LmPTR1 and are more or equally active against PTR1 than the reference 
compound 1b. The nanomolar to picomolar PTR1 inhibitors show improved selectivity for PTR1 over the off-target hDHFR by up to about 3 orders of magnitude (2c, 4e: TbPTR1 IC 50 $<0.1 \mathrm{nM}$; SI >400000) (Figure 8). The $\mathrm{IC}_{50}$ against hDHFR is typically greater than $100 \mu \mathrm{M}$, whereas inhibitory activities against TbDHFR and LmDHFR are higher. For parasite DHFR, the compounds with the best inhibitory activities have similar $\mathrm{IC}_{50}$ values to 1 b (e.g. $L \mathrm{mDHFR}$ $I_{50} 4 \mathrm{a}: 0.13 \mu \mathrm{M}, \mathbf{4 b}: 0.15 \mu \mathrm{M}$ and $\left.1 \mathrm{~b}: 0.26 \mu \mathrm{M}\right)$. Thus, the newly designed compounds show improved target inhibitory profiles, particularly for the PTR1 targets, and overall good selectivity for the parasitic proteins.

\section{The inhibitory activity against $T$. brucei is related to the hydrophobicity of the compounds}

Following the assessment of the improvement on the target inhibition level, we next determined the anti-parasitic effect on $T$. brucei brucei Lister 427 bloodstream forms and $L$. infantum intramacrophage amastigotes (Figure 10A and Table S7). The LmPTR1 and $L m D H F R$ proteins are highly similar to the corresponding $L$. infantum proteins $(91 \%$ and $96 \%$ sequence identity, respectively) but, in spite of the improved effect on both target proteins, the designed pteridines are mostly inactive against $L$. infantum. In contrast, the compounds show activity against $T$. brucei.
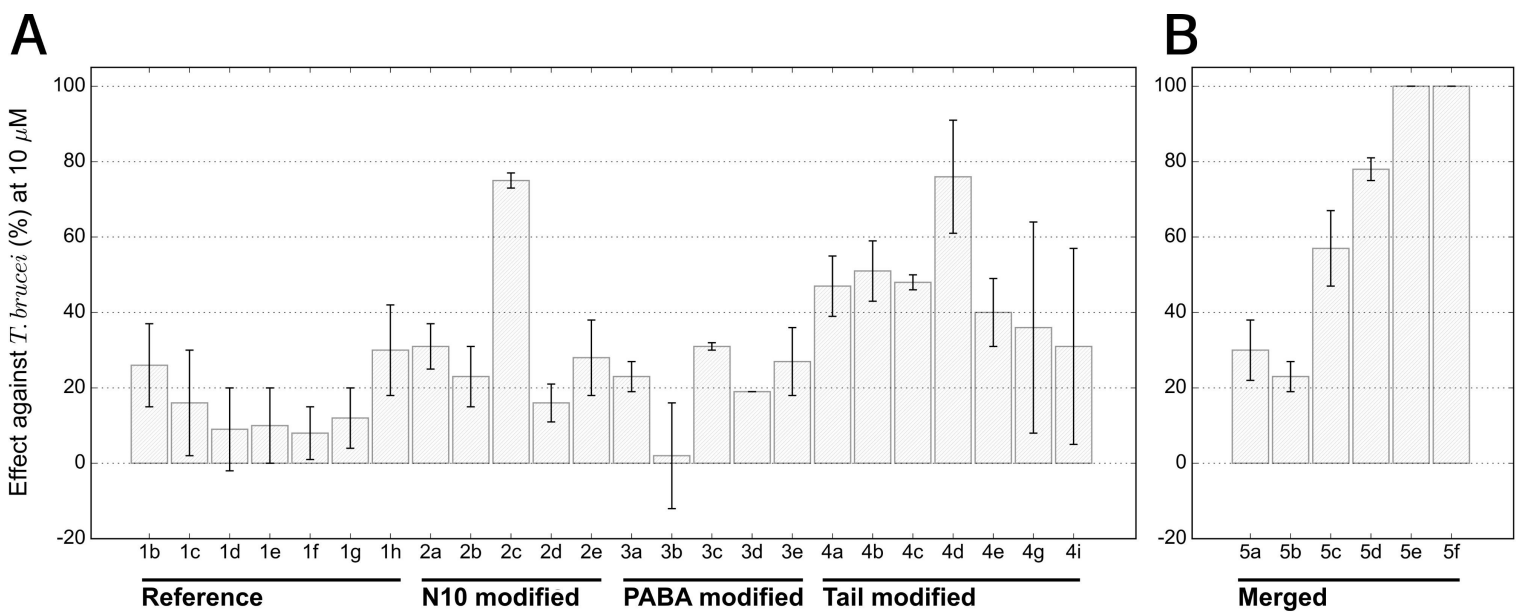

Figure 10. Anti-parasitic activity expressed as percentage of inhibition against $T$. brucei brucei for reference compounds and members of the N10-, PABA and tail-modified series (left) and the selected representatives of the merged in silico library (right). The average of at least three independent determinations is shown with the standard deviation. The inactive compounds in the tail modified series, $\mathbf{4 f}, \mathbf{4 h}$ and $\mathbf{4 j}$ were omitted. Activities can be found in Table S7.

The multiple correlation coefficient between the TbPTR1 and TbDHFR IC $\mathrm{C}_{50}$ values and the $T$. brucei bloodstream form inhibition is $\mathrm{R}=0.35$ (equation $1, \mathrm{SI}$ ), indicating that the levels of target enzyme inhibition are, for the current compounds, only weakly correlated with the exhibited anti-parasitic effect when assuming a linear correlation. PTR1 inhibition alone shows a Pearson correlation $\mathrm{R}=0.34$ with $T$. brucei inhibition, whereas $\mathrm{R}$ is only 0.24 for DHFR 
inhibition, possibly because all studied compounds are much stronger inhibitors of PTR1 than DHFR.

The low correlations between parasite and target protein inhibition can arise due to a variety of effects, such as transport issues. For example, the charged compound tail and possible polyglutamylation of the parent MTX (1a) have previously been suggested to influence compound transport. ${ }^{39,40}$ All the newly designed pteridines lack the glutamate tail, which may affect their in vivo activities, but there might be other structural or physicochemical features that render them more or less active against parasites despite similar target inhibition. For example, for $T$. brucei, we noticed that while $\mathbf{2 c}$ and $\mathbf{4 e}$ have similar effects on the targets TbPTR1 $(0.1 \mathrm{nM})$ and TbDHFR (approx. $1 \mu \mathrm{M}$ ), they differ notably in their inhibitory effect on the parasite bloodstream forms (75 vs. $40 \%$ ).

Table 1. Descriptors with significant correlations with the observed inhibitory effect on $T$. brucei for the reference compounds and pteridines of the N10-, PABA- and Tail-modified series calculated with QikProp. ${ }^{41}$ QPlogKp: Predicted skin permeability, log $\mathrm{K}_{\mathrm{p}}$; QPlogPo/w: Predicted octanol/water partition coefficient. QPlogKhsa: Prediction of binding to human serum albumin. Cohesive index: Index of cohesive interaction in solids, (no. of hydrogen bond acceptors $x$ no. of hydrogen bond donors x 0.5 / surface area) ${ }^{42}$; CIQPlogS: Conformationindependent predicted aqueous solubility, $\log S$ with $S$ in $\mathrm{mol} \mathrm{dm}^{-3}$ being the concentration of the solute in a saturated solution that is in equilibrium with the crystalline solid. $R$ (Pearson correlation) and $\mathrm{R}^{2}$ were calculated using the percentage of inhibition of the $T$. brucei brucei Lister 427 bloodstream form at $10 \mu \mathrm{M}$ compound concentration as defined in the SI. Only descriptors with at least a Pearson correlation/anti-correlation of 0.40/0.40 and two-tailed P-values lower than the chosen significance level $\alpha$ of 0.05 are reported. Covered range: property values obtained for the studied compounds. Recommended range: values the properties take for typical drug-like molecules. Resampling recovery rate indicates in how many cases (expressed as percentage) the same property was identified when leaving a single compound out of the data set. The optimization direction indicates whether higher or lower values would putatively lead to improved anti-parasitic effects.

\begin{tabular}{|l|c|c|c|c|c|}
\hline Predicted property & QPlogKp & QPlogPo/w & QPlogKhsa & Cohesive index & CIQPlogS \\
\hline $\mathbf{R}$ & 0.55 & 0.49 & 0.47 & -0.41 & -0.54 \\
\hline $\mathbf{R}^{2}$ & 0.30 & 0.24 & 0.22 & 0.17 & 0.29 \\
\hline P value & 0.003 & 0.01 & 0.01 & 0.04 & 0.004 \\
\hline $\begin{array}{l}\text { Resampling } \\
\text { recovery rate (\%) }\end{array}$ & 100 & 96 & 96 & 56 & 96 \\
\hline $\begin{array}{l}\text { Optimization } \\
\text { direction }\end{array}$ & $\uparrow$ & $\uparrow$ & $\uparrow$ & $\downarrow$ & $\downarrow$ \\
\hline Covered range & $-6.62--3.60$ & $-1.02-2.92$ & $-0.85-0.35$ & $0.02-0.04$ & $-6.71--3.19$ \\
\hline $\begin{array}{l}\text { Recommended } \\
\text { range }\end{array}$ & $-8.00--1.00$ & $-2.00-6.50$ & $-1.50-1.50$ & $0.00-0.05$ & $-6.50-0.50$ \\
\hline
\end{tabular}

Therefore, we investigated the correlations of physicochemical properties and ADMET predictors with the measured effect on T. brucei, see Table 1. Our aim was to identify which properties were indicative of a better anti-parasitic effect, possibly related to better uptake. Overall, only weak correlations of the individual properties with $T$. brucei inhibition were observed (Pearson R: 0.47-0.55 and -0.41 - -0.54; computed as defined in the SI). The strongest correlation was found for the predicted skin permeability, QPlogKp, as a descriptor linked to lipophilicity, (R: 0.55). The logPo/w and the binding to human serum albumin had 
slightly weaker correlations with the anti-parasitic effect (R: 0.49 and 0.47 , respectively). For these properties, an increase in the value corresponds with higher anti- $T$. brucei activity. In contrast, some properties showed anti-correlation, for instance, the aqueous solubility and the cohesive index ${ }^{42}$ ( $R$ : -0.54 and -0.41 , respectively). Taken together, the data indicate an improved anti-parasitic effect with increased lipophilicity of the studied compounds.

\section{Combined modifications yield pteridines with both improved target inhibition and improved anti-parasitic activity}

To explore further derivatives of the studied pteridines, we next designed a merged compound library as follows. The pteridine core scaffold was retained and the studied compounds were decomposed into fragments of their N10, PABA and Tail regions and recombined in silico in all possible combinations to yield 2014 derivatives (see SI for details). These derivatives were evaluated in docking studies against targets and off-targets and additionally prioritized by the physicochemical marker properties that showed correlations with the anti- $T$. brucei effect (Figure S9). Of the remaining 600 candidates, six were selected by expert opinion as representative compounds for synthesis and experimental evaluation (5a-5f, Figure 11).

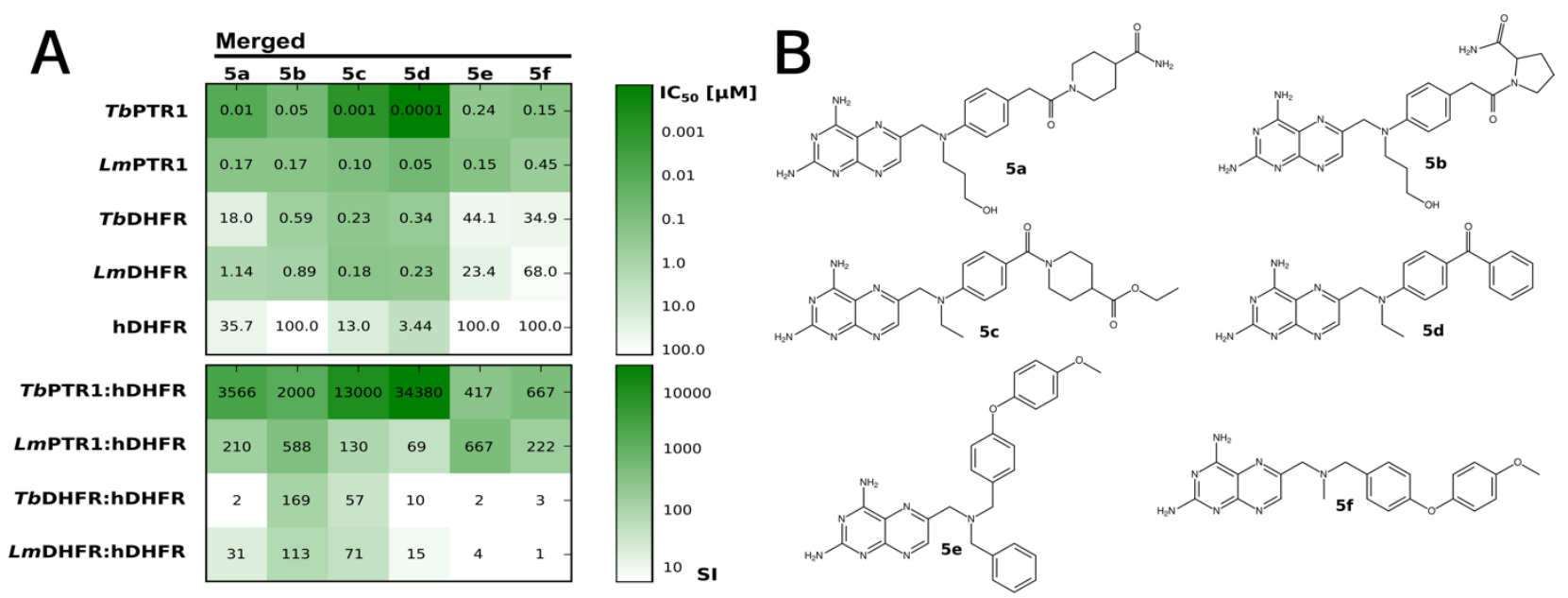

Figure 11. Inhibitory activities, selectivities and structures of the merged series of 6 pteridine derivatives. (A) The activity heatmap in the top panel shows $I_{50}$ values for the targets TbPTR1, LmPTR1, TbDHFR, LmDHFR and the off-target hDHFR. All values, as well as data for hTS, are reported in Table S1. In the bottom panel, selectivity indices are reported. (B) The structures of the selected and synthesized pteridines in the merged series.

Two compounds, $\mathbf{5 a}$ and $\mathbf{5 b}$, were chosen for their favorable interaction patterns and scores predicted by docking simulations. 5a combines the N10 hydroxypropyl fragment of $\mathbf{2 e}$, benzyl in place of the PABA phenyl of $\mathbf{3 a}$, the tail amide of reference compound $\mathbf{1 g}$, and, for $\mathbf{5 b}$, in addition, the tail pyrrolidine of reference $1 \mathrm{~h}$ replaces the tail piperidine. The activities and predicted interactions in all parasite targets are most similar to $2 \mathbf{e}$, suggesting the key importance of the hydroxy-propyl substituent to N10 for the target inhibition. Notably, while 5a is poorly selective for TbDHFR (2-fold) and modestly selective for LmDHFR ( 31 -fold), $\mathbf{5 b}$ is inactive against hDHFR, resulting in SI values of 170 and 113 for TbDHFR and LmDHFR, 
respectively. Moreover, $\mathbf{5 b}$ has $\mathrm{SI}$ values over hDHFR of about 2000 for TbPTR1 and 588 for LmPTR1. However, in contrast to most compounds, $\mathbf{5}$ b displays a weak inhibition of hTS $\left(\mathrm{IC}_{50}\right.$ $29 \mu \mathrm{M}$, Table S1).

Four additional compounds (5c-5f, Figure 11) were prioritized based on the physicochemical marker properties. Compound $\mathbf{5 c}$ combines fragments of ethyl modification to $\mathrm{N} 10$ of $\mathbf{2 a}$ and the tail ethyl ester of $\mathbf{4 a}$. Due mainly to the tail ester, this modification improves the inhibition for both TbPTR1 (IC $501 \mathrm{nM})$ and LmPTR1 (IC $\left.\mathrm{C}_{50} 0.1 \mu \mathrm{M}\right)$. The activity against TbDHFR is similar to that of the N10-modified parent $2 \mathbf{a}$, whereas $L m D H F R$ and hDHFR inhibition are again influenced by the tail modification (IC 50 LmDHFR 5c: $0.2 \mu \mathrm{M}, 4 \mathrm{a}: 0.1 \mu \mathrm{M}$; hDHFR 5c: 13 $\mu \mathrm{M}$, 4a: $12 \mu \mathrm{M}$ ). Compound $\mathbf{5 d}$ merges the ethyl $\mathrm{N} 10$ fragment of $\mathbf{2 a}$ with the unsubstituted benzene of $\mathbf{4 d}$. In TbPTR1, this boosts the nanomolar $\mathrm{IC}_{50}$ of $\mathbf{4 d}$ to the picomolar range, while the activity towards $L m P T R 1$ remains similar to $4 \mathbf{d}$. This profile can be related to the $\mathrm{N} 10$ ethyl, which seems disfavored in LmPTR1 as judged by the modest inhibition of the parent $2 \mathbf{a}\left(\mathrm{IC}_{50}\right.$ $13.3 \mu \mathrm{M})$.

Compounds $\mathbf{5 e}$ and $\mathbf{5 f}$ combine the ethylphenyl(4-methoxyphenyl) ether scaffold of $\mathbf{4} \mathbf{i}$ with the benzyl and methyl N10 modifications from 2c or 1b, respectively. Both compounds are nanomolar inhibitors of both PTR1 variants. The parent compounds, $\mathbf{2 c}$ and $\mathbf{1 b}$, inhibit the parasite DHFR variants at micromolar to submicromolar levels, while $\mathbf{4} \mathbf{i}$ is inactive against all variants of DHFR. The combination with a favorable N10 substitution is able to restore medium micromolar anti-DHFR activity for the altered scaffold of parent $4 \mathbf{i}$ in the parasite enzymes in 5e and 5f. Thus, combined N10 and tail modifications allowed for the species-specific optimization of the target inhibition profile.

Compounds $\mathbf{5 d}, \mathbf{5 e}$ and $\mathbf{5} \mathbf{f}$ show an improved percentage of $T$. brucei inhibition at $10 \mu \mathrm{M}$, in line with their selection for synthesis being motivated by altered marker properties (Figure 10B). For these compounds, $\mathrm{EC}_{50}$ values were determined, see Table 2. Indeed, the more lipophilic compounds were found to have low micromolar $\mathrm{EC}_{50} \mathrm{~S}$ against $T$. brucei brucei, with $\mathbf{5 d}$ being the best $\left(\mathrm{EC}_{50} 0.66 \pm 0.48 \mu \mathrm{M}\right)$, and they have Sls of 3-38 based on their cytotoxicity on THP-1 derived macrophages.

\section{Bulky compounds with hydrophobic substituents often display liabilities}

Potential liabilities were assessed by determining the inhibition of the $h E R G$ potassium channel, five isoforms of CYP450 (1A2, 2C9, 2C19, 2D6 and 3A4), cytotoxicity against A459 cells (human lung adenocarcinoma epithelial cell line) and mitochondrial toxiticity against 786 $\mathrm{O}$ cells (renal carcinoma cell line) for all compounds at a concentration of $10 \mu \mathrm{M}$. The results are shown in Figure 12. Further, the compounds were assessed for and passed a check for being Pan-assay interference compounds (PAINS). 
Table 2. Properties with a significant correlation with the observed inhibitory effect on $T$. brucei for compounds in the merged series calculated with QikProp. ${ }^{41}$ The properties are defined as in Table 1. Values shown in bold face are within $90 \%$ of the previously determined top value or exceeded the previously obtained range for the reference compounds and compounds in the N10-, PABA- and Tail-modified series, see Table 1. The activity against $T$. brucei brucei Lister 427 bloodstream form at $10 \mu \mathrm{M}$ compound concentration (\%inhibition) is given. For the most promising compounds, $\mathbf{5} \mathbf{d}, \mathbf{5 e}$ and $\mathbf{5 f}$, in addition, measured $\mathrm{EC}_{50}$ values, $\mathrm{CC}_{50}$ interval estimation and selectivity indices are reported and compared to pentamidine, a reference compound with activity against $T$. brucei. EC $\mathrm{C}_{50}$ represents the arithmetic average of at least two independent measurements done in triplicate. $\mathrm{CC}_{50}$ estimation was done by at least three independent cytotoxicity assessments on THP-1-derived macrophages by a colorimetric MTT (3-(4,5-dimethylthiazol-2-yl)-2,5-diphenyl tetrazolium bromide) assay, as previously reported. ${ }^{43}$ The selectivity index is determined as the $\mathrm{CC}_{50}$ or lower $\mathrm{CC}_{50}$ interval estimation divided by $\mathrm{EC}_{50}$. N.D.: Not determined.

\begin{tabular}{|c|c|c|c|c|c|c|c|c|c|}
\hline Compound & $\begin{array}{c}Q P \\
\log K p\end{array}$ & $\begin{array}{c}Q P \\
\log P o / w\end{array}$ & $\begin{array}{c}\text { QP } \\
\text { logKhsa }\end{array}$ & $\begin{array}{l}\text { Cohesive } \\
\text { index }\end{array}$ & $\begin{array}{l}\text { CIQP } \\
\text { logS }\end{array}$ & $\begin{array}{c}\text { \%inhibition } \\
\text { of } T \text {. brucei } \\
\text { at } 10 \mu \mathrm{M} \pm \\
\text { SD }\end{array}$ & $\begin{array}{c}\mathrm{EC}_{50} T \text {. brucei } \\
{[\mu \mathrm{M}] \pm \mathrm{SD}}\end{array}$ & $\mathrm{CC}_{50}[\mu \mathrm{M}]$ & $\begin{array}{l}\text { Selectivity } \\
\text { index }\end{array}$ \\
\hline $5 a$ & -6.74 & -1.16 & 0.05 & 0.04 & -4.53 & $30 \pm 8$ & N.D. & N.D. & N.D. \\
\hline $5 b$ & -6.48 & -1.32 & 0.43 & 0.04 & -6.35 & $23 \pm 4$ & N.D. & N.D. & N.D. \\
\hline $5 c$ & -5.18 & 2.02 & 0.04 & 0.03 & -5.32 & $57 \pm 10$ & N.D. & N.D. & N.D. \\
\hline $5 d$ & -3.91 & 2.19 & 0.07 & 0.02 & -5.43 & $78 \pm 3$ & $0.66 \pm 0.48$ & $25<\mathrm{CC}_{50}<50$ & 38 \\
\hline $5 e$ & -4.60 & 3.36 & -1.23 & 0.02 & -3.20 & $100 \pm 0$ & $4.53 \pm 0.42$ & $\begin{array}{c}12.5<\mathrm{CC}_{50}< \\
25\end{array}$ & 3 \\
\hline $5 f$ & -5.16 & 2.09 & -1.14 & 0.02 & -3.44 & $100 \pm 0$ & $1.30 \pm 0.05$ & $\begin{array}{c}12.5<\mathrm{CC}_{50}< \\
25\end{array}$ & 10 \\
\hline Pentamidine & N.D. & N.D. & N.D. & N.D. & N.D. & N.D. & $0.0019 \pm 0.0005$ & 10 & 5263 \\
\hline
\end{tabular}

The reference compounds and the N10 series mostly exhibit a safe profile. In contrast, aromatic modifications to the compound tail region, for instance in $\mathbf{3 d}, \mathbf{4} \mathbf{j}$ and $\mathbf{5 f}$ (PABA, Tail and Merged series, respectively) were associated with notable $h$ ERG liabilities. Increasing the hydrophobicity of the compounds further led to liabilities against some CYP isoforms, in particular, $2 \mathrm{C} 9$ and $2 \mathrm{C} 19$. The shortened tails of $3 \mathrm{c}$ and $\mathbf{3 d}$ resulted in a strong effect on CYP isoform 2D6. Finally, several of the bulky, more hydrophobic compounds resulted in a cytostatic or cytotoxic effect on A549 cells. Overall, the liability assessment suggests that increasing hydrophobicity is associated with greater compound liabilities.

In line with these observations, two of the best inhibitors of $T$. brucei bloodstream forms, $\mathbf{5 e}$ and $\mathbf{5 f}$ show $54 \%$ and $81 \%$ hERG inhibition, respectively. $\mathbf{5 e}$ and, in many cases also $\mathbf{5 f}$, affects various CYP isoforms. Finally, $\mathbf{5 f}$ is cytostatic with A549 cell growth reduced to $15 \%$ and $\mathbf{5 d}$ shows cytotoxicity, effectively completely inhibiting cell growth. Thus, the most active inhibitors of $T$. brucei bloodstream forms were found to suffer from liabilities associated with their greater hydrophobicity and would require careful optimization of their cellular specificity. 


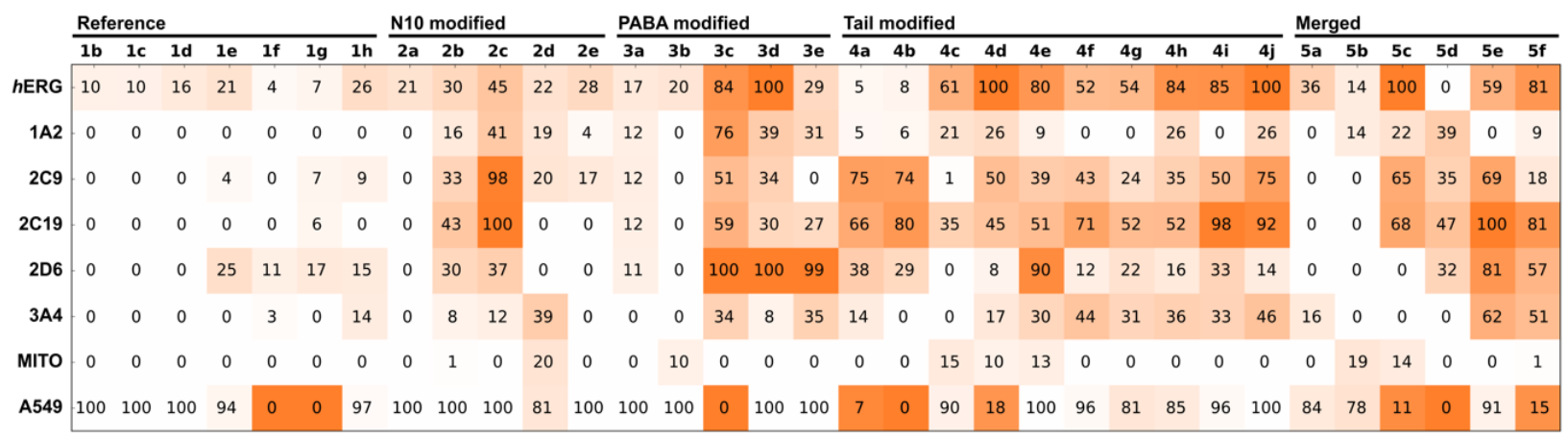

Figure 12. Heatmap representation of the liability assessment results for all the compounds studied. Inhibition of $h E R G$, five CYP isoforms (1A2, 2C9, 2C19, 2D6 and 3A4), mitochondrial toxicity (MITO) and growth inhibition of A549 cells were determined at $10 \mu \mathrm{M}$. The data are represented as percentages on a color scale from white (desired) to orange (undesired) with values reported in the map. For the inhibitory activities against $h$ ERG, CYP isoforms and mitochondrial toxicity: white $=0 \%$, orange $=100 \%$ inhibition/toxicity, while for A549 cell growth inhibition white $=100 \%$ and orange $=$ $0 \%$ growth. The values are reported in Tables $\mathbf{S 8}$ and $\mathbf{5 9 .}$

\section{Conclusions}

We applied a multitarget-based approach to the development of novel therapies for HAT and leishmaniasis, in which we focused on pteridine-based inhibitors of L. major and T. brucei PTR1 and DHFR, and successfully designed the first known picomolar inhibitors of TbPTR1. While LmPTR1/LmDHFR inhibition was previously explored for this compound class ${ }^{26}$, we here demonstrated the potential of pteridine-based inhibitors against TbPTR1 and TbDHFR. We solved a crystal structure of the reference compound $\mathbf{1 b}$ bound to TbPTR1 to confirm the overlap in observed binding modes between LmPTR1 and TbPTR1 and the preference of the methotrexate inhibitor-like bound orientation in TbPTR1. Guided by our detailed comparative study of on- and off-targets in the parasitic and human folate pathway ${ }^{31}$, crystal structures of reference compounds, and enzymatic evaluation of published reference pteridines ${ }^{26,30}$, we designed 26 new pteridine derivatives that mostly have improved activity and selectivity. For their synthesis, we made use of an advanced MW-assisted protocol to improve the reaction yield of the pteroid step with reduced reaction time compared to previous synthetic procedures. ${ }^{33}$ Further determination of the crystal structures of complexes and computational docking enabled us to obtain a complete characterization of the binding modes of the pteridines to their molecular targets and supported the derivation of a SAR. The compounds were also tested against the human off-targets hDHFR and hTS. While they were sometimes only modestly selective for the parasitic DHFR variant, many showed 1000 -fold and higher selectivities for PTR1 over the off-targets and thus, the novel PTR1 inhibitors can overall be considered selective for the parasite proteins.

While many compounds exhibited excellent inhibitory activity at the target level, they were often only modest inhibitors of $T$. brucei brucei bloodstream forms and inactive towards $L$. infantum intracellular amastigotes in vitro. We found that increased lipophilicity correlated with 
improved inhibitory effects on T. brucei. We were able to prioritize compounds for synthesis from a designed combinatorial in silico library by using predicted ADMET-related properties, which suggested a likely improvement of the trypanocidal effect. In this way, we identified three improved compounds, $\mathbf{5} \mathbf{d}, \mathbf{5 e}$ and $\mathbf{5 f}$, with low micromolar inhibition of $T$. brucei brucei $\left(\mathrm{EC}_{50} 0.66-4.53 \mu \mathrm{M}\right)$.

The modulated on-target/off-target activities and selectivities of the above compounds showed that specific combinations of the $\mathrm{N} 10$ and tail modifications allow a fine-tuning of the target inhibition profile for enzymes of specific parasite species. Furthermore, the strategy employed here of combining property prediction correlation with multitarget-based compound design was found to be a useful approach to discovering antiparasitic agents, even when the antiparasitic data are available only as a percentage of inhibition determined at a single compound concentration. Further, integration of transport-related considerations in the design ${ }^{31}$, or using, for instance, structurally related scaffolds reported in the literature, which show inhibition of the Leishmania parasite, and a similar property-based correlation concept to that presented here, may help to overcome the current limitations of the pteridine-based compounds as inhibitors of intracellular parasites. Our data show that, overall, optimization for increased lipophilicity leads to more potent pteridine-based $T$. brucei inhibitors. However, increased lipophilicity can also introduce compound liabilities, e.g. for $h E R G$ and CYPs. Strategies to avoid these, for instance by making use of a similar property-based optimization strategy, should thus be incorporated in future design efforts.

\section{Experimental Procedures}

General Synthesis Information. Reagent grade chemicals and solvents were purchased from commercial suppliers and used without further purification. Reactions were monitored by TLC on silica gel plates (Kieselgel 60, F254, Merck) and visualized using UV light, cerium ammonium sulphate or alkaline $\mathrm{KMnO}_{4}$ aqueous solution. Solvents are abbreviated as follows: tetrahydrofuran (THF), ethyl ether $\left(\mathrm{Et}_{2} \mathrm{O}\right)$, dimethyl sulfoxide (DMSO), dimethylacetamide (DMA), ethyl acetate (EtOAc), dichloromethane (DCM), dimethyl formamide (DMF), methanol $(\mathrm{MeOH})$ and acetonitrile (ACN). The structures of the isolated compounds were confirmed by NMR spectroscopy and mass spectrometry. NMR spectra were recorded on Bruker 400 and 600 spectrometers with ${ }^{1} \mathrm{H}$ at $400.134-600 \mathrm{MHz}$ and ${ }^{13} \mathrm{C}$ at $100.62-151 \mathrm{MHz}$. The purity of all synthesized compounds was determined by elemental analyses performed on a PerkinEImer 240C instrument and by measurement of the melting point interval on a Stuart SMP3 instrument. Liquid chromatography-mass spectrometry (LC-MS) was used to obtain the molecular mass and analyze the purity of the products. The specifications of the LC-MS instrument were the following: Agilent 1200 HPLC coupled to a 6120 
quadrupole mass spectrometer (ESI-API), UV detection at 254 and $210 \mathrm{~nm}$, Agilent Zorbax XDB-18 $\mathrm{C}_{18}$ column $(50 \times 4.6 \mathrm{~mm}, 3.5 \mu \mathrm{m})$, gradient mobile phase consisting of $\mathrm{MeOH} /$ water $/ 0.1 \%$ formic acid buffer, and a flow rate of $1.00 \mathrm{~mL} / \mathrm{min}$.

\section{General synthetic procedure A: Reductive alkylation of amines using nitriles (32-} $33,51,74)$. After two vacuum $/ \mathrm{H}_{2}$ cycles to remove air from the reaction tube, the stirred mixture of the amine (1.0 equiv.), PdIC catalyst (10 wt \% of the amine), the respective $\mathrm{RCN}$ (5.0 equiv.) and $\mathrm{NH}_{4} \mathrm{OAc}$ (1.0 equiv.) in $\mathrm{MeOH}(5.0 \mathrm{~mL})$ was hydrogenated under ambient pressure (balloon) at room temperature (rt) for the appropriate time (24-36 h). The reaction mixture was filtrated using Celite cake before the filtrate was concentrated under reduced pressure. The residue was partitioned between $\mathrm{Et}_{2} \mathrm{O}(10 \mathrm{~mL})$ and water $(10 \mathrm{~mL})$. The aqueous phase was extracted thrice with $\mathrm{Et}_{2} \mathrm{O}(10 \mathrm{~mL})$, and the combined organic phases were washed with brine $(10 \mathrm{~mL})$, dried with anhydrous $\mathrm{Na}_{2} \mathrm{SO}_{4}$, filtered and concentrated under reduced pressure to yield the amines without further purification.

General synthetic procedure B: Amide coupling reaction for the synthesis of 2728, 40-49, 52-54, 56-57, 71-72, 75. Carboxylic acid compounds (1 equiv.), EDC. HCl (1.1 equiv.) and HOBt ( 0.1 equiv.) were added to a dried round-bottomed flask and dissolved in DMF dry under $\mathrm{N}_{2}$. The reaction mixture was cooled down to $0^{\circ} \mathrm{C}$ and stirred for $30 \mathrm{~min}$ before adding it to the respective amine (1 equiv.) with/without TEA (2-3 equiv.). After stirring overnight at $\mathrm{rt}$, the mixture was washed $1 \mathrm{x}$ with saturated $\mathrm{NaHCO}_{3}, 1 \mathrm{x}$ with $\mathrm{H}_{2} \mathrm{O}$ and $1 \mathrm{x}$ with brine. The washed organic mixture was then dried with $\mathrm{Na}_{2} \mathrm{SO}_{4}$, concentrated in vacuo and purified using column chromatography $\left(\mathrm{SiO}_{2}\right.$, eluent: $\mathrm{Cy} / \mathrm{EtOAc}$ or $\mathrm{DCM} / \mathrm{MeOH}$ or $\mathrm{DCM} / \mathrm{EtOAc/MeOH}$ ) to give the desired amide.

General synthetic procedure C: MW alkylation 1b-c, 2a-e, 3a-c, 4a-j, 5a-f. To a suspension of amine intermediates (1 equiv.) in DMA (3 $\mathrm{mL})$ in a microwave Biotage vial, 29 (1.2 equiv.), $\mathrm{K}_{2} \mathrm{CO}_{3}$ (3 equiv.) and $\mathrm{KI}$ (0.1 equiv.) were added. The vial was sealed and heated by microwave irradiation in a Biotage $\AA$ Initiator + microwave at $60^{\circ} \mathrm{C}$ for $20 \mathrm{~min}$ (30' for compounds $\mathbf{3 c}, \mathbf{4} \mathbf{d}$ and $\mathbf{5 d}$ ), before cooling to $\mathrm{rt}$ and diluting with water $(20 \mathrm{~mL})$. The precipitate was then collected by filtration and dried before the final compound was purified by fractional crystallization from methanol, DCM and $\mathrm{Et}_{2} \mathrm{O}$.

General synthetic procedure D: $S_{N} A r$ for the preparation of 4-substituted benzaldehyde (63-65). A mixture of substituted phenol 58-60 (1 equiv.), 4fluorobenzaldehyde (1 equiv.), and $\mathrm{K}_{2} \mathrm{CO}_{3}$ (3 equiv.) in DMF (10 mL) was refluxed for 
16-18 $\mathrm{h}$ under nitrogen. After cooling, the solution was concentrated in vacuo to give a crude residue, which was purified by crystallization in $1 \mathrm{~N} \mathrm{NaHCO}_{3}$. The obtained crystal was washed with $\mathrm{H}_{2} \mathrm{O}$ to obtain the desired benzaldehyde derivatives.

\section{General synthetic procedure E: Preparation of primary amines from 4-substituted} benzaldehyde (66-68). A solution of carbonyl (aldehyde) compounds 66-68 (1 equiv.) and hydroxylammonium chloride (1.2 equiv.) in ethanol $(30 \mathrm{~mL})$ was stirred for $1 \mathrm{~h}$ at $\mathrm{rt}$. Subsequently, $12 \mathrm{M}$ hydrochloric acid (4 equiv.) and zinc dust (2.5 equiv.) were slowly added to the solution and let to stir at rt for $15 \mathrm{~min}$. To the resulting slurry, a solution of ammonia $(30 \%, 14 \mathrm{~mL})$ and sodium hydroxide $(6 \mathrm{M}, 30 \mathrm{~mL})$ was added dropwise and the mixture was stirred at $\mathrm{rt}$ for another $15 \mathrm{~min}$. Then, the resulting solution was extracted with DCM, dried over anhydrous $\mathrm{Na}_{2} \mathrm{SO}_{4}$, and filtered. The solvent was removed under vacuum to give the amines without further purification.

Protein expression and purification. Recombinant TbPTR1, LmPTR1, TbDHFR-TS, LmDHFR-TS, hDHFR and hTS were expressed and purified according to previously reported procedures. ${ }^{26,43,44}$

Crystallization of TbPTR1 and LmPTR1. Well-ordered monoclinic crystals of histidinetagged TbPTR1 were obtained by the vapor diffusion hanging drop technique at $\mathrm{rt}^{45}$ Drops were prepared by mixing equal volumes of protein and precipitant solution (2 $2.5 \mathrm{M}$ sodium acetate and $0.1 \mathrm{M}$ sodium citrate $\mathrm{pH} 5$ ) according to a previously described procedure. ${ }^{43}$ The TbPTR1-cofactor-inhibitor ternary complexes were obtained by the soaking technique. The compounds, solubilized in DMSO, were diluted in the cryoprotectant solution ( $30 \% \mathrm{vol} / \mathrm{vol}$ glycerol added to the precipitant solution) to a final concentration of 2-4 mM (keeping the DMSO concentration below $10 \% \mathrm{vol} / \mathrm{vol}$ ). Crystals were then transferred in the resulting soaking/cryoprotectant solution and flash frozen in liquid nitrogen after 8-24 h exposure.

Crystals of $L m P T R 1$ were prepared as described elsewhere. ${ }^{38}$ The LmPTR1-cofactor$2 \mathrm{e}$ ternary complex was obtained by the soaking technique, adding $2 \mathrm{mM}$ compound (solubilized in DMSO, without exceeding the 10\% drop volume) directly into the crystallization drop. After $5 \mathrm{~h}$, crystals were transferred to the cryoprotectant solution and flash frozen in liquid nitrogen.

Data collection, structure solution and refinement. X-ray crystallographic data were collected using synchrotron radiation at the Diamond Light Source (DLS, Didcot, United 
Kingdom) beamlines $104-1$ and 103 equipped with a Dectris Pilatus 6M-F and a Pilatus3 $6 \mathrm{M}$ detector, respectively. Reflections were integrated using MOSFLM and scaled with Scala (CCP4 suite). ${ }^{46-50}$ Data collection and processing statistics are reported in Table S2. The crystals of TbPTR1 and LmPTR1 belonged to the primitive monoclinic space group $\mathrm{P} 2{ }_{1}$ and the primitive orthorhombic space group $\mathrm{P} 2{ }_{1} 2_{1} 2_{1}$, respectively. Both had a functional enzyme tetramer in the asymmetric unit. The structures were solved by molecular replacement using MOLREP and either a TbPTR1 (PDB-ID 5jdc) or a LmPTR1 tetramer (PDB-ID 5I4n) as the searching model (all non-protein atoms were excluded). ${ }^{38,43,51}$ Models were refined using REFMAC5 (CCP4 suite). ${ }^{52}$ Visual inspection and manual rebuilding of missing atoms was performed using Coot. ${ }^{53,54}$ Water molecules were added with the automated standard procedures implemented in the software ARP/wARP and checked with Coot. ${ }^{55}$ In the higher resolution complexes of TbPTR1 with compounds $\mathbf{2 a}$ and $\mathbf{2 e}$, all atoms were refined anisotropically in the final refinement cycles and hydrogen atoms were added in the calculated positions. The occupancies of exogenous ligands were individually adjusted to values resulting in atomic displacement parameters comparable to those of surrounding protein atoms in fully occupied sites. The final models were checked with Coot and Procheck. ${ }^{56}$ Statistics for data refinement are reported in Table S3. Figures were generated using CCP4mg. ${ }^{57}$ Coordinates and structure factors were deposited in the Protein Data Bank under the PDB-IDs 6rx5 (TbPTR1-NADPH/NADP $\left.{ }^{+}-1 \mathbf{b}\right), \quad 6 r \times 0 \quad$ (TbPTR1NADPH/NADP $\left.{ }^{+}-\mathbf{2 a}\right), \quad 6 r \times 6 \quad\left(\right.$ TbPTR1-NADPH/NADP $\left.{ }^{+}-\mathbf{2 e}\right)$, and $6 r \times c \quad$ (LmPTR1$\left.\mathrm{NADPH} / \mathrm{NADP}^{+} \mathbf{- 2 e}\right)$.

TbPTR1, TbDHFR, LmPTR1, LmDHFR, hDHFR and hTS target/off-target enzyme assays. In vitro assays for TbPTR1 and LmPTR1 were based on the coupled assay reported by Shanks et al. ${ }^{58}$ The assay non-enzymatically links the reduction of cytochrome $c$ (Cc) with the reduction of dihydrobiopterin to tetrahydrobiopterin, catalyzed by PTR1. The formation of reduced $\mathrm{Cc}\left(\mathrm{Fe}^{2+}\right)$ results in a signal increase in the photometric readout at $550 \mathrm{~nm}$ wavelength. TbPTR1 and LmPTR1 assays were performed in a buffer containing $20 \mathrm{nM}$ sodium citrate $(\mathrm{pH}$ 6.0) in a well-plate-based format as previously reported. ${ }^{43}$ LmDHFR, TbDHFR, hDHFR and hTS activities were assessed spectrophotometrically according to published procedures. ${ }^{59,60}$ Each inhibitory compound was assayed at five different concentrations in duplicate (confidence interval $\rho<0.05)$ and $\mathrm{IC}_{50}$ values were calculated as described in the SI. 


\section{Computational preparation of pteridine compounds and protein receptors and SiteMap}

calculation of DHFR pocket volumes. The 3D structures of the reference and designed compounds were generated from SMILES strings and optimized with the OPLS_2005 force field using LigPrep of Maestro (Schrödinger, LLC) as described previously, except that tautomers were created for the $\mathrm{pH}$ range 5.0-8.0 and both $\mathrm{N} 1$-deprotonated and $\mathrm{N} 1$ protonated tautomers were considered for every compound. ${ }^{43,61-64}$ In addition, all different substituents to the N10 position, PABA modifications and compound tail alterations present in compounds $\mathbf{1 b}-\mathbf{4} \mathbf{j}$ were combined in all possible permutations in silico in a 'merged' series and prepared similarly.

All receptors were prepared in the presence of MTX (from the following PDB-IDs for TbPTR1: 2c7v, LmPTR1: 1e7w, TbDHFR and LmDHFR: 3cl9 and hDHFR: 1u72) to improve the interactions of binding site residues and the conserved water molecules with the pteridine core. Receptor preparation was following published procedures with minor modifications. ${ }^{43,61,62,65-67}$ For the LmPTR1 (PDB-ID 1e92) and TbPTR1 (PDB-ID $2 \times 9 \mathrm{~g}$ ) receptors, an energy minimization with a harmonic restraint of $25 \mathrm{kcal} \mathrm{mol}^{-1} \AA^{-2}$ on heavy atoms and no restraint on hydrogens was performed until the heavy atom RMSD relative to the previous minimization step was less than $0.30 \AA .{ }^{68}$ For the TbDHFR receptor, PDB-ID 3rg9 was used; for LmDHFR, our previously published homology model based on a TCDHFR-TS template (PDB-ID 3inv) was chosen. ${ }^{31}$ For off-target docking, we used the hDHFR structure 1u72. For PTR1, we also considered the previously described set of conserved water molecules identified by a WatCH clustering approach. ${ }^{43,65}$ Further, using $\mathrm{WatCH}$, we identified conserved water sites in hDHFR as described in the SI. ${ }^{65}$ Except for the parasite DHFR variants, where the identification of a conserved water set was not possible, all receptors were prepared both with the identified set of conserved structural waters and without explicit water molecules. Grid preparation was done as described before for $L m P T R 1$ and TbPTR $1^{43}$ with the following grid centers and rotatable groups: (i) LmPTR1: center Phe113, rotatable OH in Ser111, Thr184, Tyr191, Tyr194, Thr195, Tyr283 and NADP ${ }^{+}$ribose; (ii) TbPTR1: center Phe97, rotatable OH/SH in Ser95, Cys168, Tyr174 and NADP ${ }^{+}$ribose; (iii) LmDHFR: center Phe31, rotatable OH/SH in Thr35, Thr36, Ser61, Cys130, Tyr137, Thr155, NADP ${ }^{+}$ribose; (iv) TbDHFR: center Phe58, rotatable $\mathrm{OH}$ in Thr46, Thr62, Thr86, Ser89, Ser98, Tyr166, Thr184, NADP ${ }^{+}$ribose; and (v) hDHFR: center Phe34, rotatable $\mathrm{OH}$ in Thr38, Thr39, Ser59, Tyr121, $\mathrm{NADP}^{+}$ribose.

The volumes of the binding pockets of TbDHFR (PDB-ID 3rg9), hDHFR (1u72) and the LmDHFR homology model were computed with Schrödinger SiteMap ${ }^{61,69,70}$ as described in the SI. 
Computational docking studies. Docking studies were performed using a rigid receptor in Glide standard precision (SP) and extra precision (XP) modes and employing the Induced Fit (IF) protocol to allow for refinement of binding site residues. ${ }^{61,71-77}$ For rigid receptor docking, van der Waals radii scaling of ligand atoms and settings for sampling, addition of Epik state penalties to the docking score, rewarding of intramolecular hydrogen bonds and enhancement of the planarity of conjugated $\pi$-groups were chosen as described previously ${ }^{43}$, but a total of 50 poses per ligand were subjected to post-docking energy minimization. For the in silico library, we used SP docking with a constraint on all heavy atoms of the pteridine core to match the orientation of MTX in the corresponding protein receptor with a tolerance of $1 \AA$.

In addition, since some compounds showed major variation in substituent size when compared to the starting scaffold and explicit water molecules are treated as frozen in the standard SP/XP docking, additional studies allowing protein side chain and water reorganization in response to ligand binding were performed using the standard protocol for the IF workflow implemented in Maestro. The planarity of conjugated $\pi-$ groups was enhanced and a Prime refinement was performed for residue side chains within $5 \AA$ of ligand atoms. XP redocking was done as previously described, yielding up to 20 receptor-ligand complexes per compound. ${ }^{43}$

The validation of the docking protocol is presented in the SI.

\section{Computational property prediction, Pan-assay interference compounds (PAINS) and} correlation analysis with anti-parasitic data. Physico-chemical descriptors and parameters related to ADMET were computed for all prepared compounds using QikProp (Schrödinger). ${ }^{41}$ Pearson correlations $(R), R^{2}$ values and two-tailed P-values for each property with the measured percentage of inhibition of $T$. brucei at $10 \mu \mathrm{M}$ compound concentration were computed using SciPy and Python scripts written for the purpose. Only properties with a Pvalue equal to or below the statistical significance level $\alpha=0.05$ were considered further. To ensure robustness, a resampling analysis was performed by leaving every compound out once before recomputing the correlations. Properties with $\mathrm{R}>0.40$ or $<-0.40$ and $\mathrm{P}$-value $\leq \alpha$ in $>$ $50 \%$ of the resampling correlation analyses were considered to be the most robust markers for the optimization for anti-parasitic effect. These properties were employed to prioritize compounds for synthesis as part of the Merged series, for details, see SI and Fig. S9.

In addition, a multivariate correlation coefficient between parasite target protein inhibition and anti-parasitic activity was computed, for details see SI.

Finally, all synthesized compounds were checked for PAINS filters A, B and C, undesirable substructure moieties, covalent inhibition, and compliance with the rule-of- 
five with the FAF-Drugs4 webserver (fafdrugs4.mti.univ-paris-diderot.fr/) by inputing SMILES strings for the compounds. ${ }^{78}$

In vitro biological evaluation against $T$. brucei and $L$. infantum intramacrophage amastigotes. The efficacy against $T$. brucei brucei Lister 427 bloodstream forms was evaluated in a modified resazurin-based assay as previously described. ${ }^{79}$ Cells were grown at $37^{\circ} \mathrm{C}$ and $5 \% \mathrm{CO}_{2}$ in a complete $\mathrm{HMI}-9$ medium supplemented with $10 \%$ fetal calf serum (FCS) and $100 \mathrm{UI} / \mathrm{mL}$ of penicillin/streptomycin. Cultures were then diluted to a cell density of $2 \times 10^{6}$ $/ \mathrm{mL}$. For the assay, compounds were prepared in $10 \mathrm{mM} \mathrm{DMSO}$ and diluted in HMI-9 to a 40 $\mu \mathrm{M}$ solution ( $0.4 \% \mathrm{DMSO})$. The assay solution was further used to perform serial dilutions (1:2) in a 96-well plate. Mid-log bloodstream forms (100 $\mu \mathrm{L})$ were added in complete HMI-9 medium at a final cell density of $1 \times 10^{4} / \mathrm{mL}$ in a well volume of $200 \mu \mathrm{L}$ after compound addition, leading to a maximum DMSO concentration of $0.2 \%$. Following incubation for $72 \mathrm{~h}$ at $37^{\circ} \mathrm{C}$ and $5 \% \mathrm{CO}_{2}, 20 \mu \mathrm{L}$ of $0.5 \mathrm{mM}$ resazurin solution were added and plates were further incubated for $4 \mathrm{~h}$ under similar conditions. Fluorescence was then measured using a Synergy 2 multimode reader (BioTek) at 540 and $620 \mathrm{~nm}$ excitation and emission wavelength, respectively. The efficacy of compounds against $L$. infantum intracellular amastigotes was determined according to Sereno et al. with slight modifications described in detail in the $\mathrm{SI}^{80}$

Liability assays. The $h E R G$ cardiotoxicity assay was performed using the Invitrogen Predictor $h E R G$ fluorescence polarization (FP) assay. A membrane fraction containing $h E R G$ (Predictor $h$ ERG membrane) was used together with a red fluorescent high-affinity ligand of the $h$ ERG channel (Predictor $h$ ERG Tracer Red). Displacement of the latter from $h$ ERG by binding of the test compound can be determined in a FP-based format. ${ }^{43}$

Cytochrome P450 (CYP450) assays against isoforms 1A2, 2C9, 2C19, 2D6 and 3A4 were performed using the Promega P450-Glo assay platform. Microsomal preparations of cytochrome P450s from baculovirus-infected insect cells were used. In this assay, light is generated when a CYP450 enzyme acts on its substrate and a decrease thereof was indicative of inhibitory effects of the tested compound on the respective isoform. ${ }^{43}$ For monitoring mitochondrial toxicity caused by the test compounds in the 786-O cell line, uptake of MitoTracker Red (chloromethyl-X-rosamine) combined with high content imaging was used. Cells were maintained in Rosswell Park Memorial Institute (RPMI)1640 medium containing $2 \mathrm{mM}$ glutamine, FCS $(10 \% \mathrm{v} / \mathrm{v})$, streptomycin $(100 \mu \mathrm{g} / \mathrm{mL})$, and penicillin $\mathrm{G}(100 \mathrm{U} / \mathrm{mL}){ }^{43}$

The cytotoxicity assay against A549 cells was performed using the CellTiter-Glo assay from Promega. The number of viable cells present is directly proportional to the cellular ATP content, which is detected. The A549 cells were obtained from DSMZ (German 
Collection of Microorganisms and Cell Cultures, Braunschweig, Germany) and grown in Dulbecco's modified Eagle medium (DMEM) with FCS (10\% v/v), streptomycin (100 $\mu \mathrm{g} / \mathrm{mL})$ and penicillin $\mathrm{G}(100 \mathrm{U} / \mathrm{mL}){ }^{43}$

\section{Supplemental Information}

Supplemental Figures S1-9, Supplemental Tables S1-12, Supplemental experimental procedures and compound characterization (pdf), NMR spectra of compounds (pdf), SMILES and activities of compounds (csv).

Additional supplementary data are freely available at https://fairdomhub.org/investigations/417: QikProp prediction results for synthesized and in silico pteridines and corresponding SOP. PAINS filtering results, Python modules for correlating QikProp data with experimental activities and for computing a multiple correlation between target and parasite inhibition. Compound library construction data and SOP, prepared docking receptors (PDB) with SOP, all Glide XP rigid-body docking results as PDBfiles of the receptor-ligand complexes and SOP as well as selected discussed induced fit docking results and corresponding SOP.

\section{PDB-Codes}

Crystal structures described in this paper are available in the Protein Data Bank with identifiers: 6rx5 (TbPTR1-NADPH/NADP ${ }^{+}$-1b), $6 r x 0$ (TbPTR1-NADPH/NADP ${ }^{+}$-2a), 6rx6 (TbPTR1-NADPH/NADP $\left.{ }^{+}-2 \mathbf{e}\right), 6 r x c\left(L m P T R 1-N A D P H / N A D P^{+}-2 e\right)$.

\section{Corresponding author information}

* Email: mariapaola.costi@unimore.it (M.P.C.), a.venturelli@tydockpharma.com (A.V.), rebecca.wade@h-its.org (R.C.W.)

\section{Author Contributions}

+ These authors contributed equally and are considered as first co-authors. Conceptualization, M.P.C., A.V., R.C.W.; Computational Methodology and Investigation, I.P., J. P.-H.; Chemical synthesis Methodology and Investigation, A.Q.; Enzyme assay Methodology and Investigation, R.L., M.S., P.L.; Crystallography Methodology and Investigation, G.L., F.D.P., L.D.I., C.P.; ADMET Methodology and Investigation, S.G., G.W., B.E., M.K.; Parasite assay Methodology and Investigation, N.S.; Writing - Original Draft, I.P., 
Writing - Review \& Editing, I.P., J. P.-H., M.P.C., R.C.W.; Supervision, S.M., A.C.S., M.P.C, A.V., R.C.W.

\section{Notes}

The authors declare no competing interests.

\section{Acknowledgements}

This work has received funding from the European Union's Seventh Framework Programme for research, technological development, and demonstration under grant agreement no. 603240 (NMTrypl, New Medicines for Trypanosomatidic Infections, https://fp7-nmtrypi.eu/). We thank Prof. Antonio Carta, University of Sassari, for providing the reference compounds 1d-1h. I.P., J.P.-H. and R.C.W. gratefully acknowledge the support of the Klaus Tschira Foundation. J.P.-H. acknowledges support from the Polish National Science Centre (grant no. 2016/21/D/NZ1/02806), the BIOMS program at the Interdisciplinary Center for Scientific Computing (IWR), Heidelberg University, and the Interdisciplinary Centre for Mathematical and Computational Modelling (ICM), University of Warsaw (grant no. G70-13, GB70-11, GA73-25).

\section{Abbreviations used}

DHFR: Dihydrofolate reductase; HAT: Human African Trypanosomiasis; MTX: Methotrexate; NTDs: Neglected Tropical Diseases; PABA: para-amino benzoic acid; PAINS: Pan-Assay Interference compounds; PTR1: Pteridine reductase 1; SI: Selectivity index; TS: Thymidylate synthase

\section{References}

1. Neglected tropical diseases. https://www.who.int/neglected diseases/en/ (accessed May 2020).

2. The WHO Strategic and Technical Advisory Group for Neglected Tropical Diseases (WHO STAG). https://www.who.int/neglected diseases/diseases/Adoption additional NTDs.pdf?ua=1, $\% 202017$.

3. Barrett, M. P., Burchmore, R. J. S., Stich, A., Lazzari, J. O., Frasch, A. C., Cazzulo, J. J., Krishna, S. The trypanosomiases. Lancet. 2003 362, 1469-1480.

4. Blum, J., Schmid, C., Burri, C. Clinical aspects of 2541 patients with second stage human African trypanosomiasis. Acta Trop. 2006 97, 55-64.

5. Stuart, K., Brun, R., Croft, S., Fairlamb, A., Gürtler, R. E., McKerrow, J., Reed, S., Tarleton, R. Kinetoplastids: related protozoan pathogens, different diseases. J. Clin. Invest. 2008 118, 1301-1310.

6. Cunningham, A. C. Parasitic adaptive mechanisms in infection by Leishmania. Exp. Mol. 
Pathol. 2002 72, 132-141.

7. Herwaldt, B. L. Leishmaniasis. Lancet. 1999 354, 1191-1199.

8. Castillo, E., Dea-Ayuela, M. A., Bolás-Fernández, F., Rangel, M., González-Rosende, M. E. The kinetoplastid chemotherapy revisited: current drugs, recent advances and future perspectives. Curr. Med. Chem. 2010 17, 4027-4051.

9. Machado-Silva, A., Goulart Guimarães, P. P., Pereira Tavares, C. A., Sinisterra, R. D. New perspectives for leishmaniasis chemotherapy over current anti-leishmanial drugs: a patent landscape. Expert Opin. Ther. Pat. 2015 25, 247-260.

10. Gilbert, I. H. Drug discovery for neglected diseases: molecular target-based and phenotypic approaches. J. Med. Chem. 2013 56, 7719-7726.

11. Müller, J., Hemphill, A. Drug target identification in protozoan parasites. Expert Opin. Drug Discov. 2016 11, 815-824.

12. Borsari, C., Quotadamo, A., Ferrari, S., Venturelli, A., Cordeiro-da-Silva, A., Santarem, N., Costi, M. P. Chapter Two - Scaffolds and biological targets avenue to fight against drug resistance in leishmaniasis. Annu. Rep. Med. Chem. 2018 51, 39-95.

13. Shuvalov, O., Petukhov, A., Daks, A., Fedorova, O., Vasileva, E., Barlev, N. A. One-carbon metabolism and nucleotide biosynthesis as attractive targets for anticancer therapy. Oncotarget 2017 8, 23955-23977.

14. Anderson, A. C., Wright, D. L. Antifolate agents: a patent review (2010 - 2013). Expert Opin. Ther. Pat. 2014 24, 687-697.

15. Hawser, S., Lociuro, S., Islam, K. Dihydrofolate reductase inhibitors as antibacterial agents. Biochem. Pharmacol. 2006 71, 941-948.

16. Yuthavong, Y., Yuvaniyama, J., Chitnumsub, P., Vanichtanankul, J., Chusacultanachai, S., Tarnchompoo, B., Vilaivan, T., Kamchonwongpaisan, S. Malarial (Plasmodium falciparum) dihydrofolate reductase-thymidylate synthase: structural basis for antifolate resistance and development of effective inhibitors. Parasitology 2005 130, 249-259.

17. Christensen, K. E., MacKenzie, R. E. Mitochondrial one-carbon metabolism is adapted to the specific needs of yeast, plants and mammals. Bioessays 2006 28, 595-605.

18. Cullia, G., Tamborini, L., Conti, P., De Micheli, C., Pinto, A. Folates in Trypanosoma brucei: Achievements and opportunities. ChemMedChem 2018 13, 2150-2158.

19. Bello, A. R., Nare, B., Freedman, D., Hardy, L., Beverley, S. M. PTR1: A reductase mediating salvage of oxidized pteridines and methotrexate resistance in the protozoan parasite Leishmania major. Proc. Natl. Acad. Sci. U. S. A. 1994 91, 11442-11446.

20. Dawson, A., Gibellini, F., Sienkiewicz, N., Tulloch, L. B., Fyfe, P. K., McLuskey, K., Fairlamb, A. H., Hunter, W. N. Structure and reactivity of Trypanosoma brucei pteridine reductase: inhibition by the archetypal antifolate methotrexate. Mol. Microbiol. 2006 61, 1457-1468.

21. Vickers, T. J., Beverley, S. M. Folate metabolic pathways in Leishmania. Essays Biochem. $201151,63-80$.

22. Ong, H. B., Sienkiewicz, N., Wyllie, S., Fairlamb, A. H. Dissecting the metabolic roles of pteridine reductase 1 in Trypanosoma brucei and Leishmania major. J. Biol. Chem. 2011 286, 10429-10438.

23. Sienkiewicz, N., Ong, H. B., Fairlamb, A. H. Trypanosoma brucei pteridine reductase 1 is essential for survival in vitro and for virulence in mice. Mol. Microbiol. 2010 77, 658-671.

24. Mpamhanga, C. P., Spinks, D., Tulloch, L. B., Shanks, E. J., Robinson, D. A., Collie, I. T., Fairlamb, A. H., Wyatt, P. G., Frearson, J. A., Hunter, W. N., Gilbert, I. H., Brenk, R. One scaffold, three binding modes: Novel and selective pteridine reductase 1 inhibitors derived from fragment hits discovered by virtual screening. J. Med. Chem. 2009 52, 4454-4465.

25. Spinks, D., Ong, H. B., Mpamhanga, C. P., Shanks, E. J., Robinson, D. A., Collie, I. T., Read, K. D., Frearson, J. A., Wyatt, P. G., Brenk, R., Fairlamb, A. H., Gilbert, I. H. Design, synthesis and biological evaluation of novel inhibitors of Trypanosoma brucei pteridine reductase 1. ChemMedChem 2011 6, 302-308.

26. Cavazzuti, A., Paglietti, G., Hunter, W. N., Gamarro, F., Piras, S., Loriga, M., Allecca, S., Corona, P., McLuskey, K., Tulloch, L., Gibellini, F., Ferrari, S., Costi, M. P. Discovery of potent pteridine reductase inhibitors to guide antiparasite drug development. Proc. Natl. 
Acad. Sci. U. S. A. 2008 105, 1448-1453.

27. Ivanetich, K. M., Santi, D. V. Bifunctional thymidylate synthase-dihydrofolate reductase in protozoa. FASEB J. 1990 4, 1591-1597.

28. Schormann, N., Senkovich, O., Walker, K., Wright, D. L., Anderson, A. C., Rosowsky, A., Ananthan, S., Shinkre, B., Velu, S., Chattopadhyay, D. Structure-based approach to pharmacophore identification, in silico screening, and three-dimensional quantitative structure-activity relationship studies for inhibitors of Trypanosoma cruzi dihydrofolate reductase function. Proteins 2008 73, 889-901.

29. Schormann, N., Velu, S. E., Murugesan, S., Senkovich, O., Walker, K., Chenna, B. C., Shinkre, B., Desai, A., Chattopadhyay, D. Synthesis and characterization of potent inhibitors of Trypanosoma cruzi dihydrofolate reductase. Bioorg. Med. Chem. 2010 18, 4056-4066.

30. Corona, P., Gibellini, F., Cavalli, A., Saxena, P., Carta, A., Loriga, M., Luciani, R., Paglietti, G., Guerrieri, D., Nerini, E., Gupta, S., Hannaert, V., Michels, P. A. M., Ferrari, S., Costi, P. M. Structure-based selectivity optimization of piperidine-pteridine derivatives as potent Leishmania pteridine reductase inhibitors. J. Med. Chem. 2012 55, 8318-8329.

31. Panecka-Hofman, J., Pöhner, I., Spyrakis, F., Zeppelin, T., Di Pisa, F., Dello lacono, L., Bonucci, A., Quotadamo, A., Venturelli, A., Mangani, S., Costi, M. P., Wade, R. C. Comparative mapping of on-targets and off-targets for the discovery of antitrypanosomatid folate pathway inhibitors. Biochim. Biophys. Acta, Gen. Subj. 2017 1861, 3215-3230.

32. Tulloch, L. B., Martini, V. P., Iulek, J., Huggan, J. K., Lee, J. H., Gibson, C. L., Smith, T. K., Suckling, C. J., Hunter, W. N. Structure-based design of pteridine reductase inhibitors targeting African sleeping sickness and the leishmaniases. J. Med. Chem. 2010 53, 221229.

33. Quotadamo, A., Linciano, P., Costi, M. P., Venturelli, A. Optimization of $N$-alkylation in the synthesis of methotrexate and pteridine-based derivatives under microwave-irradiation. Chemistry Select 2019 4, 4429-4433.

34. Sajiki, H., Ikawa, T., Hirota, K. Reductive and catalytic monoalkylation of primary amines using nitriles as an alkylating reagent. Org. Lett. 2004 6, 4977-4980.

35. Ikawa, T., Fujita, Y., Mizusaki, T., Betsuin, S., Takamatsu, H., Maegawa, T., Monguchia, Y., Sajiki, H. Selective N-alkylation of amines using nitriles under hydrogenation conditions: facile synthesis of secondary and tertiary amines. Org. Biomol. Chem. 2012 10, 293-304.

36. Ayedi, M. A., Le Bigot, Y., Ammar, H., Abid, S., El Gharbi, R., Delmas, M. Synthesis of primary amines by one-pot reductive amination of aldehydes. Synth. Commun. 2013 43, 2127-2133.

37. Gourley, D. G., Schüttelkopf, A. W., Leonard, G. A., Luba, J., Hardy, L. W., Beverley, S. M., Hunter, W. N. Pteridine reductase mechanism correlates pterin metabolism with drug resistance in trypanosomatid parasites. Nat. Struct. Biol. 2001 8, 521-525.

38. Di Pisa, F., Landi, G., Dello lacono, L., Pozzi, C., Borsari, C., Ferrari, S., Santucci, M., Santarem, N., Cordeiro-da-Silva, A., Moraes, C. B., Alcantara, L. M., Fontana, V., FreitasJunior, L. H., Gul, S., Kuzikov, M., Behrens, B., Pöhner, I., Wade, R. C., Costi, M. P., Mangani, S. Chroman-4-one derivatives targeting pteridine reductase 1 and showing antiparasitic activity. Molecules 2017 22, 426.

39. Synold, T. W., Willits, E. M., Barredo, J. C. Role of folylpolyglutamate synthetase (FPGS) in antifolate chemotherapy; a biochemical and clinical update. Leuk Lymphoma, 1996, 21, 9-15.

40. Gibson, M. W., Dewar, S., Ong, H. B., Sienkiewicz, N., Fairlamb, A. H. Trypanosoma brucei DHFR-TS revisited: characterisation of a bifunctional and highly unstable recombinant dihydrofolate reductase-thymidylate synthase. PLoS Negl Trop Dis, 2016, 10 , e0004714.

41. Schrödinger, LLC, New York, NY, Schrödinger Release 2015-4, 2015, QikProp v4.6.

42. Jorgensen, W. L., Duffy, E. M. Prediction of drug solubility from Monte Carlo simulations. Bioorg. Med. Chem. Lett. 2000 10, 1155-1158. 
43. Borsari, C., Luciani, R., Pozzi, C., Poehner, I., Henrich, S., Trande, M., Cordeiro-da-Silva, A., Santarem, N., Baptista, C., Tait, A., Di Pisa, F., Dello lacono, L., Landi, G., Gul, S., Wolf, M., Kuzikov, M., Ellinger, B., Reinshagen, J., Witt, G., Gribbon, P., Kohler, M., Keminer, O., Behrens, B., Costantino, L., Tejera Nevado, P., Bifeld, E., Eick, J., Clos, J., Torrado, J., Jiménez-Antón, M. D., Corral, M. J., Alunda, J. M., Pellati, F., Wade, R. C., Ferrari, S., Mangani, S., Costi, M. P. Profiling of flavonol derivatives for the development of antitrypanosomatidic drugs. J. Med. Chem. 2016 59, 7598-7616.

44. Cardinale, D., Guaitoli, G., Tondi, D., Luciani, R., Henrich, S., Salo-Ahen, O. M., Ferrari, S., Marverti, G., Guerrieri, D., Ligabue, A., Frassineti, C., Pozzi, C., Mangani, S., Fessas, D., Guerrini, R., Ponterini, G., Wade, R. C., Costi, M. P. Protein-protein interface-binding peptides inhibit the cancer therapy target human thymidylate synthase. Proc. Natl. Acad. Sci. U. S. A. 2011 108, E542-E549.

45. Benvenuti, M., Mangani, S. Crystallization of soluble proteins in vapor diffusion for X-ray crystallography. Nat. Protoc. 2007 2, 1633-1651.

46. Battye, T. G. G., Kontogiannis, L., Johnson, O., Powell, H. R., Leslie, A. G. W. iMOSFLM: a new graphical interface for diffraction-image processing with MOSFLM. Acta Crystallogr. 2011 67, 271-281.

47. Powell, H. R., Johnson, O., Leslie, A. G. W. Autoindexing diffraction images with iMosflm. Acta Crystallogr. 2013 69, 1195-1203.

48. Evans, P. Scaling and assessment of data quality. Acta Crystallogr. 2006 62, 72-82.

49. Evans, P. An introduction to data reduction: space-group determination, scaling and intensity statistics. Acta Crystallogr. 2011 67, 282-292.

50. CCP4. The CCP4 suite: programs for protein crystallography. Acta Crystallogr. 199450 , 760-763.

51. Vagin, A., Teplyakov, A. Molecular replacement with MOLREP. Acta Crystallogr. 2010 66, 22-25.

52. Murshudov, G. N., Skubák, P., Lebedev, A. A., Pannu, N. S., Steiner, R. A., Nicholls, R. A., Winn, M. D., Long, F., Vagin, A. A. REFMAC5 for the refinement of macromolecular crystal structures. Acta Crystallogr. 2011 67, 355-367.

53. Emsley, P., Cowtan, K. Coot: model-building tools for molecular graphics. Acta Crystallogr. 2004 60, 2126-2132.

54. Emsley, P., Lohkamp, B., Scott, W. G., Cowtan, K. Features and development of Coot. Acta Crystallogr. 2010 66, 486-501.

55. Langer, G. G., Cohen, S. X., Lamzin, V. S., Perrakis A. Automated macromolecular model building for X-ray crystallography using ARP/wARP version 7. Nat. Protoc. 2008 3, 11711179.

56. Laskowski, R. A., MacArthur, M. W., Thornton, J. M. Validation of protein models derived from experiment. Curr. Opin. Struct. Biol. 1998 8, 631-639.

57. Potterton, L., McNicholas, S., Krissinel, E., Gruber, J., Cowtan, K., Emsley, P., Murshudov, G. N., Cohen, S., Perrakis, A., Noblec, M. Developments in the CCP4 molecular-graphics project. Acta Crystallogr. 2004 60, 2288-2294.

58. Shanks, E. J., Ong, H. B., Robinson, D. A., Thompson, S., Sienkiewicz, N., Fairlamb, A. H., Frearson, J. A. Development and validation of a cytochrome $c$-coupled assay for pteridine reductase 1 and dihydrofolate reductase. Anal. Biochem. 2010 396, 194-203.

59. Ferrari, S., Morandi, F., Motiejunas, D., Nerini, E., Henrich, S., Luciani, R., Venturelli, A., Lazzari, S., Calò, S., Gupta, S., Hannaert, V., Michels, P. A. M., Wade, R. C., Costi, M. P. Virtual screening identification of nonfolate compounds, including a CNS drug, as antiparasitic agents inhibiting pteridine reductase. J. Med. Chem. 2011 54, 211-221.

60. Tan, X., Huang, S., Ratnam, M., Thompson, P. D., Freisheim, J. H. The importance of loop region residues $40-46$ in human dihydrofolate reductase as revealed by site-directed mutagenesis. J. Biol. Chem. 1990 265, 8027-8032.

61. Schrödinger, LLC, New York, NY, Schrödinger Release 2015-4, 2015, LigPrep v3.6; Epik v3.4; Protein Preparation Wizard (PrepWizard); SiteMap v3.7; Glide v6.9; Induced Fit Docking Protocol, Prime v4.2.

62. Linciano, P., Dawson, A., Pöhner, I., Costa, D. M., Sá, M. S., Cordeiro-da-Silva, A., 
Luciani, R., Gul, S., Witt, G., Ellinger, B., Kuzikov, M., Gribbon, P., Reinshagen, J., Wolf, M., Behrens, B., Hannaert, V., Michels, P. A. M., Nerini, E., Pozzi, C., Di Pisa, F., Landi, G., Santarem, N., Ferrari, S., Saxena, P., Lazzari, S., Cannazza, G., Freitas-Junior, L. H., Moraes, C. B., Pascoalino, B. S., Alcântara, L. M., Bertolacini, C. P., Fontana, V., Wittig, U., Müller, W., Wade, R. C., Hunter, W. N., Mangani, S., Costantino, L., Costi, M. P. Exploiting the 2-Amino-1,3,4-thiadiazole scaffold to inhibit Trypanosoma brucei pteridine reductase in support of early-stage drug discovery. ACS Omega 2017 2, 5666-5683.

63. Shelley, J. C., Cholleti, A., Frye, L. L., Greenwood, J. R., Timlin, M. R., Uchimaya, M. Epik: a software program for $\mathrm{pK}_{\mathrm{a}}$ prediction and protonation state generation for drug-like molecules. J. Comput. Aided Mol. Des. 2007 21, 681-691.

64. Greenwood, J. R., Calkins, D., Sullivan, A. P., Shelley, J. C. Towards the comprehensive, rapid, and accurate prediction of the favorable tautomeric states of drug-like molecules in aqueous solution. J. Comput. Aided Mol. Des. 2010 24, 591-604.

65. Sanschagrin, P. C., Kuhn, L. A. Cluster analysis of consensus water sites in thrombin and trypsin shows conservation between serine proteases and contributions to ligand specificity. Protein Sci. 1998 7, 2054-2064.

66. Sastry, G. M., Adzhigirey, M., Day, T., Annabhimoju, R., Sherman, W. Protein and ligand preparation: parameters, protocols, and influence on virtual screening enrichments. J. Comput. Aided Mol. Des. 2013 27, 221-234.

67. Li, H., Robertson, A. D., Jensen, J. H. Very fast empirical prediction and rationalization of protein pKa values. Proteins 2005 61, 704-721.

68. Banks, J. L., Beard, H. S., Cao, Y., Cho, A. E., Damm, W., Farid, R., Felts, A. K., Halgren, T. A., Mainz, D. T., Maple, J. R., Murphy, R., Philipp, D. M., Repasky, M. P., Zhang, L. Y., Berne, B. J., Friesner, R. A., Gallicchio, E., Levy, R. M. Integrated Modeling Program, Applied Chemical Theory (IMPACT). J. Comput. Chem. 2005 26, 1752-1780.

69. Halgren, T. New method for fast and accurate binding-site identification and analysis. Chem. Biol. Drug Des. 2007 69, 146-148.

70. Halgren, T. A. Identifying and characterizing binding sites and assessing druggability. J. Chem. Inf. Model. 2009 49, 377-389.

71. Friesner, R. A., Banks, J. L., Murphy, R. B., Halgren, T. A., Klicic, J. J., Mainz, D. T., Repasky, M. P., Knoll, E. H., Shelley, M., Perry, J. K., Shaw, D. E., Francis, P., Shenkin, P. S. Glide: a new approach for rapid, accurate docking and scoring. 1. Method and assessment of docking accuracy. J. Med. Chem. 2004 47, 1739-1749.

72. Halgren, T. A., Murphy, R. B., Friesner, R. A., Beard, H. S., Frye, L. L., Pollard, W. T., Banks, J. L. Glide: a new approach for rapid, accurate docking and scoring. 2. Enrichment factors in database screening. J. Med. Chem. 2004 47, 1750-1759.

73. Friesner, R. A., Murphy, R. B., Repasky, M. P., Frye, L. L., Greenwood, J. R., Halgren, T. A., Sanschagrin, P. C., Mainz, D. T. Extra Precision Glide: Docking and scoring incorporating a model of hydrophobic wnclosure for protein-ligand complexes. J. Med. Chem. 2006 49, 6177-6196.

74. Sherman, W., Beard, H. S., Farid, R. Use of an induced fit receptor structure in virtual screening. Chem. Biol. Drug Des. 2006 67, 83-84.

75. Sherman, W., Day, T., Jacobson, M. P., Friesner, R. A., Farid, R. Novel procedure for modeling ligand/receptor induced fit effects. J. Med. Chem. 2006 49, 534-553.

76. Jacobson, M. P., Pincus, D. L., Rapp, C. S., Day, T. J. F., Honig, B., Shaw, D. E., Friesner, R. A. A hierarchical approach to all-atom protein loop prediction. Proteins 2004 55, 351367.

77. Jacobson, M. P., Friesner, R. A., Xiang, Z., Honig, B. On the role of the crystal environment in determining protein side-chain conformations. J. Mol. Biol. 2002 320, 597-608.

78. Lagorce, D., Sperandio, O., Baell, J. B., Miteva, M. A., Villoutreix, B. O. FAF-Drugs3: a web server for compound property calculation and chemical library design. Nucleic Acids Res. 2015 43, W200-207. https://fafdrugs4.mti.univ-paris-diderot.fr (accessed October 2020).

79. Bowling, T., Mercer, L., Don, R., Jacobs, R., Nare, B. Application of a resazurin-based high-throughput screening assay for the identification and progression of new treatments 
for human African trypanosomiasis. Int. J. Parasitol. Drugs Drug Resist. 2012 2, 262-270. 80. Sereno, D., Cavaleyra, M., Zemzoumi, K., Maquaire, S., Ouaissi, A., Lemesre, J. L. Axenically grown amastigotes of Leishmania infantum used as an in vitro model to investigate the pentavalent antimony mode of action. Antimicrob. Agents Chemother. 1998 42, 3097-3102.

Table of Contents Graphic

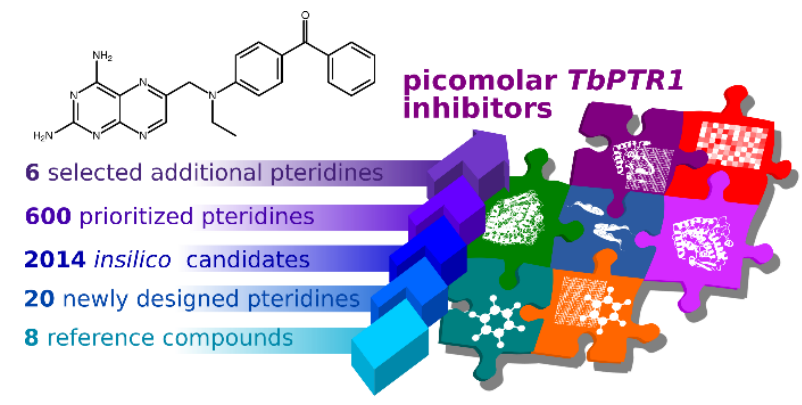

